\title{
Estimated Population Near Uranium Tailings
}

\author{
C. H. Bloomster \\ D. R. Brown \\ G. A. Bruno \\ S. N. Craig \\ J. A. Dirks \\ E. A. Griffin \\ J. W. Reis \\ J. K. Young
}

January 1984

Prepared for the U.S. Environmental Protection Agency under Related Services Agreement with the U.S. Department of Energy Contract DE-AC06-76RLO 1830

Pacific Northwest Laboratory Operated for the U.S. Department of Energy by Battelle Memorial Institute 


\title{
DISCLAIMER
}

This report was prepared as an account of work sponsored by an agency of the United States Government. Neither the United States Government nor any agency thereof, nor any of their emplcyees, makes any warranty, express or implied, or assumes any legal liability or responsibility for the accuracy, completeness, or usefulness of any information, apparatus, product, or process disclosed, or represents that its use would not infringe privately owned rights. Reference herein to any specific commercial product, process, or service by trade name, trademark, manufacturer, or otherwise, does not necessarily constitute or imply its endorsement, recommendation, or favoring by the United States Government or any agency thereof. The views and opinions of authors expressed herein do not necessarily state or reflect those of the United States Government or any agency thereof.

\author{
PACIFIC NORTHWEST LABORATORY \\ operated by \\ BATTELLE \\ for the \\ UNITED STATES DEPARTMENT OF ENERGY \\ under Contract DE-AC06-76RLO 1830
}

Printed in the United States of America
Available from
National Technical Information Service
United States Department of Commerce
5285 Port Royal Road
Springfield, Virginia 22161
NTIS Price Codes
Microfiche A01
Printed Copy
Pages
$001-025$
$026-050$


PNL-4959

UC-70

ESTIMATED POPULATION NEAR

URANIUM TAILINGS
C. H. Bloomster
J. A. Dirks
D. R. Brown
E. A. Griffin
G. A. Bruno
J. W. Reis
S. N. Craig
J. K. Young

January 1984

Prepared for the U.S. Environmental Protection Agency under Related Services Agreement

with the U.S. Department of Energy

Contract DE-AC06-76RLO 1830

Pacific Northwest Laboratory

Richland, Washington 99352 


\section{CONTENTS}

\begin{tabular}{|c|c|c|c|c|c|c|c|c|c|c|c|c|c|}
\hline INTRODUCTION & - & - & $\cdot$ & - & $\cdot$ & $\cdot$ & $\because$ & $\cdot$ & • & • & - & - & 1 \\
\hline PRE-TRIP PRE & PARAT & ION & - & - & $\cdot$ & $\cdot$ & $\cdot$ & $\cdot$ & $\cdot$ & $\bullet^{\circ}$ & - & • & - \\
\hline ON-SITE DATA & COLL & ECTION & - & $\cdot$ & • & $\cdot$ & $\cdot$ & $\cdot$ & • & $\bullet^{\circ}$ & - & • & - \\
\hline ANALYSIS OF F & F IELD & DATA & • & - & • & $\cdot$ & $\cdot$ & • & • & $\bullet$ & • & • & \\
\hline RESULTS • & - & - & • & - & • & $\cdot$ & $\cdot$ & $\cdot$ & $\cdot$ & $\bullet$ & • & • & - \\
\hline REFERENCES & - & - & - & - & - & $\cdot$ & - & $\bullet$ & $\cdot$ & 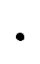 & $\bullet$ & & \\
\hline APPENDIX A - & MILL & SITE $P$ & POP & ATION & & & - & • & - & • & $\bullet$ & & \\
\hline
\end{tabular}




\section{.}


INTRODUCTION

The U.S. Environmental Protection Agency (EPA) was directed by Congress to promulgate health and environmental standards for uranium mill tailings under the Uranium Mill Tailings Radiation Control Act of 1978. This Act established two programs to protect public health, safety and the environment from uranium mill tailings. One program was aimed at sites designated inactive--sites at which all milling had stopped and which are no longer under license. The second program was directed at active sites--sites that are licensed by the Nuclear Regulatory Commission (NRC) or the state in which the site is located. EPA issued such standards during 1983.

Estimates of the health risks from radon emitted from tailings sites were a most important consideration in developing these standards. Because radon emitted from tailings piles disperses rapidly in the atmosphere, risk estimates are greatly dependent on the total number of people living nearby and on their proximity to the tailings areas. Thus, it is necessary to have accurate population data at tailings sites to make reasonably accurate estimates of the health risk.

The only population data available to EPA were those contained in environmental impact statements for active sites and in technical reports prepared throughout 1981 by Ford, Bacon and Davis Utah, Inc. for inactive sites. However, the information in these reports was sparse, inconsistent, and lacked sufficient detail for use in assessing health risks. Therefore, EPA concluded that better population estimates were needed to assure reasonably accurate health risk estimates. This led to the Pacific Northwest Laboratory (PNL) surveys described in this report.

The 27 active and 25 inactive mill sites that were to be investigated by PNL are shown in Table 1 and Table 2, respectively. As can be seen in Table 1 , those sites considered active may be either operating or shut-down. Eight PNL personnel performed the population studies, which took place during the months of April, May, and June in 1983. For each mill site, a table showing population by radius $(1 / 2,1,2,3,4$, and 5 kilometers) in 16 compass directions was generated. These tables appear in Appendix $A$. 
TABLE 1. Active U.S. Uranium Mills

Operating Mills (16)

Uravan, Colorado

Canon City, Colorado

Milan, New Mexico

Ambrosia Lake, New Mexico (Kerr-McGee Nuclear)

Panna Maria, Texas

Blanding, Utah

La Sal, Utah

Moab, Utah

Ford, Washington

Gas Hills, Wyoming (Pathfinder Mines)

Gas Hills, Wyoming (Union Carbide)

Powder River, Wyoming (Rocky Mountain Energy)

Powder River, Wyoming (Exxon Minerals)

Red Desert, Wyoming

Shirley Basin, Wyoming (Pathfinder Mines)

Shirley Basin, Wyoming (Petrotomics)

Shut-Down Mills (11)

Bluewater, New Mexico

Seboyeta, New Mexico

Church Rock, New Mexico

Marquez, New Mexico

Edgemont, South Dakota

Falls City, Texas (Conoco-Pioneer Nuclear)

Ray Point, Texas

Hanksville, Utah

Wellpinit, Washington

Jeffrey City, Wyoming

Gas Hills, Wyoming (Federal-American Partners) 
TABLE 2. Inactive U.S. Uranium Mills

Inactive Mills (25)

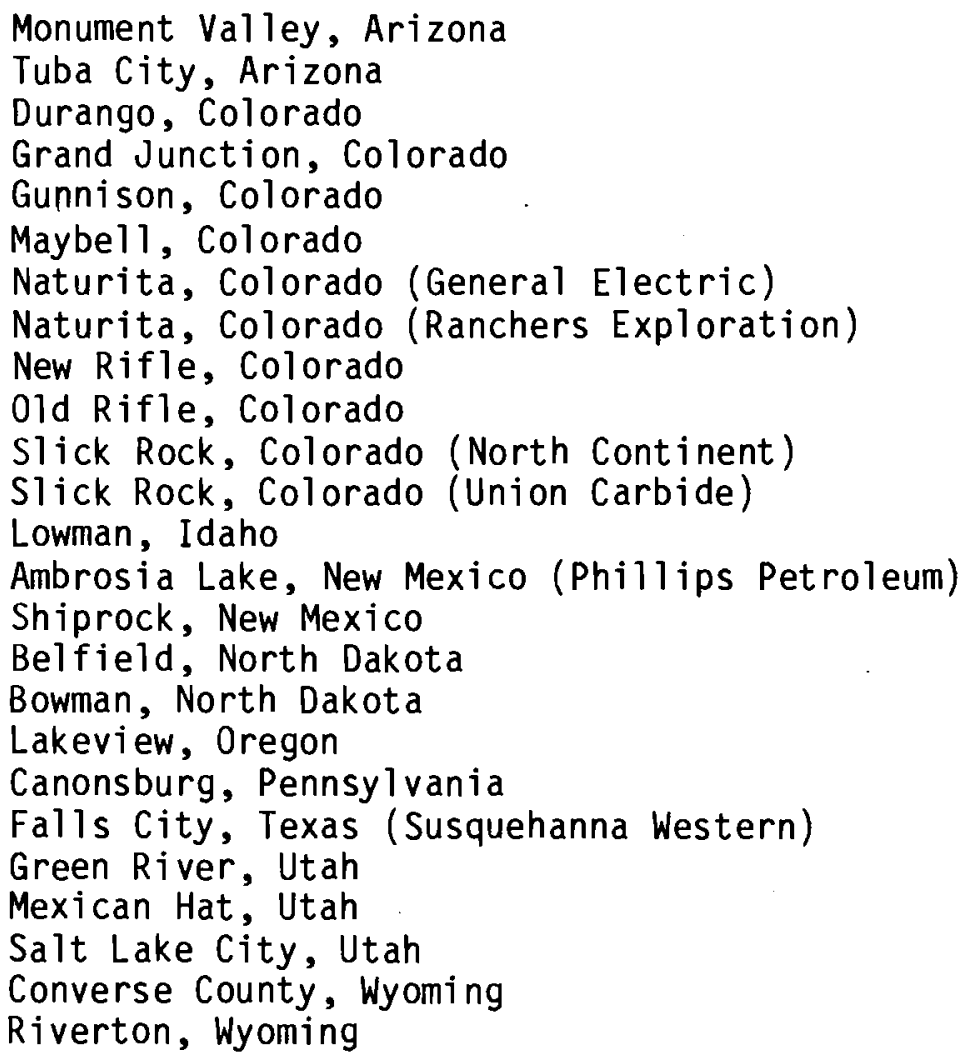




\section{PRE-TRIP PREPARATION}

The first step undertaken was to gather information on the 52 uranium mill sites through available literature sources. The primary objective was to find tailings layout diagrams and coordinates, but data concerning mill operation, any previous population surveys, and other background information were also gathered.

In general, much more information was available for inactive uranium mills than for operating or shut-down mills. Perhaps the most valuable source identified was a series of engineering assessments of tailings piles at inactive mill sites prepared throughout 1981 by Ford, Bacon and Davis Utah, Inc. (1-20) Another useful source was a report having gamma radiation surveys at inactive uranium mill sites that was prepared in August 1975 by EPA. (21)

Information for operating and shut-down sites was found in a report prepared in April 1982 by Pacific Northwest Laboratory for NRC. (22) This report discusses the environmental factors affecting stabilization of radon suppression covers for uranium mill tailings and also provides location maps. Also of use for these types of mill sites were various environmental impact statements filed by the operating companies.

After these literature sources were reviewed, each mill location was plotted on a general U.S. road map. Mill sites were then grouped and assigned to team members as efficiently as possible, making every effort to minimize traveling distances between sites, hence minimizing travel costs. Ten such groups were formed with a range of 3 to $7 \mathrm{mill}$ sites per group. Eight PNL personnel performed the site visits, since 2 people were assigned 2 groups each.

U.S.G.S. topographic quadrangle maps were used as base plotting maps for the population field surveys. Researchers referred to a topographic index for their particular state to identify the $71 / 2$ minute topographic quadrangles necessary to cover the area around their assigned mills. However, many of the mills were located in areas where only 15 minute topographic maps were available. Maps were ordered if their receipt could be guaranteed prior to the researcher's departure; otherwise, they were purchased when the researcher arrived at his destination. 
If maps were received prior to departure, the tailings layout, the tailings centroid, and the corresponding 5 kilometer radius circle around the centroid were penciled in. In many cases, however, no adequate tailings layout diagrams could be found in the literature. This was especially true for the operating mill sites.

Some individuals contacted state, county, and city planning offices before they left to obtain information over the phone or to arrange meetings upon their arrival. Others waited until they arrived before establishing these contacts. 


\section{ON-SITE DATA COLLECTION}

After the preliminary collection of maps and site information, team members began on-site data collection. Once in the field, appropriate visits were made to the various planning agencies to secure any additional maps, aerial photos, or statistical population data to be used during the site surveys. Topographic maps with plotted mill tailings disposal areas were the basis for beginning the on-site population surveys.

For most mills, enough detailed information was available to accurately locate and plot the tailings disposal areas for the mill site before beginning the field surveys. For some mills, however, site coordinates and detailed mill tailings layouts were unavailable. In these cases, the information was obtained from on-site inspections of the mill facilities. This visual siting technique was difficult in some instances due to the large extent of companyowned area around the mills. In these instances, tailings disposal areas were sighted at a distance and sketched as best as possible on the topographic maps.

Multiple tailings areas existed on many sites. To facilitate a speedy population density count, an approxmiate center of tailings was selected and a 5 kilometer radius circle was penciled on the maps. To insure adequate ground coverage due to the approximated centers, an additional $1 / 2$ to $1 \mathrm{kilometer}$ area peripheral to the 5 kilometer radius was surveyed.

The actual field surveys involved counting occupied dwellings and plotting their locations on the topographic maps described earlier. Dwelling counts were made by visual inspection via automobile. Only public roads were traversed, so as not to trespass on private lands. Some difficulty arose in navigating and plotting when using older topographic maps that did not show new road changes. In some cases, especially in the sparsely populated and isolated areas, occupied dwellings were difficult to sight due to lengthy private access roads marked no trespassing or to inclement weather causing impassable roads. In other areas, it was difficult to determine if dwellings were occupied due to a seasonal-type appearance or if a "for sale" sign appeared. Under these circumstances, possible clues as to habitation that were used included: a car in front of the dwelling, toys or tools in the yard, clothes hanging on an outside 
line, a usable looking mailbox, a recently traveled access road, or smoke coming from the dwelling's chimney.

Densely populated areas posed greater difficulties in estimating occupied dwellings. In these areas, such as near Salt Lake City, Utah, Grand Junction, Colorado, and Canonsburg, Pennsylvania, statistical sampling techniques were used. Areas within the $5 \mathrm{~km}$ study radius were selected at random for detailed on-site field surveys. These results were later combined with other detailed census tract and block data to derive the estimated population numbers. 


\section{ANALYSIS OF FIELD DATA}

Each team member analyzed their plotted field maps and their recorded field data to derive a population distribution around each mill site. The same techniques and standards were followed for each site to insure consistency of results.

First, the centroid of the tailings area was accurately determined for each mill site. In the case of multiple tailings areas, each individual area was assigned a centroid. In conjunction with a coordinate system, each individual centroid was weighted according to its area to derive the appropriate "overal1" centroid. A transparent template with inscribed radii at 1/2, 1, 2, 3 , 4 and 5 kilometers and divisions at 16 compass directions was used to accurately and uniformly divide the site into sectors. Plotted occupied dwellings were summed in each sector and tabulated.

Overlapping sites occurred in some cases. When this happened, area was apportioned to each site to avoid double counting of population. Straight line divisions were drawn through the points of intersection of the radi $i$ around each site center. In the final tabulated population results, asterisks were used to indicate when such site areas fell within other site boundaries. No double counting occurs at any adjacent mill sites.

In determining the population figures for each mill site, the number of occupied dwellings in each sector was multiplied by the average number of persons per household for each particular county. These county statistics for 1980 were obtained from the U.S. Bureau of Census and are shown in Table 3 . In a few cases, the average number of persons per household for each city was used because it was considered more representative and often more recent than 1980 county averages. City averages that were used are shown in Table 4.

Because it was not feasible to count every occupied dwelling at the Canonsburg, Pennsylvania, Grand Junction, Colorado, and Salt Lake City, Utah mill sites, the above method of population determination could not be used. For chese densely populated sites, estimation techniques using detailed 1980 U.S. Bureau of Census tract and block data were employed. The sectors formed 
by the radii and divisions at 16 compass directions were drawn on census tract or census block maps. Population within each census tract or census block was apportioned to each sector on an area basis if the distribution of population appeared relatively uniform. If information gathered in the field or through aerial photographs showed the distribution of population within each census tract or census block to be non-uniform, the population was apportioned according to these observations. 
TABLE 3. County Averages - Number of Persons Per Household

\begin{tabular}{|c|c|c|}
\hline State & County & Average Persons/Household \\
\hline Arizona & $\begin{array}{l}\text { Apache } \\
\text { Coconino }\end{array}$ & $\begin{array}{l}4.09 \\
3.15\end{array}$ \\
\hline Colorado & $\begin{array}{l}\text { Fremont } \\
\text { Garfield } \\
\text { Gunnison } \\
\text { La P P lat a } \\
\text { Montrose } \\
\text { San Migue } 1\end{array}$ & $\begin{array}{l}2.59 \\
2.72 \\
2.47 \\
2.70 \\
2.84 \\
2.55\end{array}$ \\
\hline I daho & Boise & 2.71 \\
\hline New Mexico & $\begin{array}{l}\text { Mck inl ey } \\
\text { San Juan } \\
\text { Val encia }\end{array}$ & $\begin{array}{l}3.73 \\
3.24 \\
3.17\end{array}$ \\
\hline North Dakota & $\begin{array}{l}\text { Billings } \\
\text { Bowman } \\
\text { Stark }\end{array}$ & $\begin{array}{l}3.10 \\
2.77 \\
2.91\end{array}$ \\
\hline Oregon & Lake & 2.68 \\
\hline South Dakota & Fall River & 2.53 \\
\hline Texas & $\begin{array}{l}\text { Karnes } \\
\text { Live Oak } \\
\text { Wilson }\end{array}$ & $\begin{array}{l}2.96 \\
2.88 \\
3.06\end{array}$ \\
\hline Utah & $\begin{array}{l}\text { Emery } \\
\text { Garfield } \\
\text { Grand } \\
\text { San Juan }\end{array}$ & $\begin{array}{l}3.48 \\
3.00 \\
2.98 \\
4.04\end{array}$ \\
\hline Washington & $\begin{array}{l}\text { Lincoln } \\
\text { Stevens }\end{array}$ & $\begin{array}{l}2.56 \\
2.92\end{array}$ \\
\hline Wyoming & $\begin{array}{l}\text { Carbon } \\
\text { Converse } \\
\text { Fremont } \\
\text { Natrona } \\
\text { Sweetwater }\end{array}$ & $\begin{array}{l}2.85 \\
2.99 \\
2.98 \\
2.75 \\
2.95\end{array}$ \\
\hline
\end{tabular}


TABLE 4. City Averages - Number of Persons Per Household

Average Persons/Household

Gunnison, Colorado

$3.02(1)$

Rifle, Colorado

$2.70^{(2)}$

Houston, Pennsylvania

$2.35^{(3)}$

Chartier Township North Strabane Township Pennsylvania
Cecil Township

$2.92^{(3)}$

(1) 1981 city survey.

(2) 1983 city survey.

(3) 1980 U.S. Bureau of Census. 


\section{RESULTS}

Estimated total population within a 5 kilometer radius of the centroid of the tailings area is shown for active mill sites in Table 5, and for inactive mill sites in Table 6 . As evidenced by the tables, total population observed varied significantly, ranging from 7 sites having zero population to the Salt Lake City, Utah site having an estimated total population of 91,498 within the defined 5 kilometer radius.

The distribution of these total population numbers for each of the 52 sites can be found in the tables in Appendix A. Each table gives precise longitudinal and latitudinal coordinates of the centroid of the tailings area, as well as the number of people in each sector formed by transposing 16 compass point directions upon concentric rings of $1 / 2,1,2,3,4$ and 5 kilometers around the tailing centroid. These tables appear in the order shown in Tables 5 and 6. 
TABLE 5. Estimated Total Population Within 5 Kilometers of Active Uranium Mills

\section{Operating Mills (16)}

Uravan, Colorado

Canon City, Colorado

Milan, New Mexico

Ambrosia Lake, New Mexico (Kerr-McGee Nuclear)

Panna Maria, Texas

Blanding, Utah

La Sal, Utah

Moab, Utah

Ford, Washington

Gas Hills, Wyoming (Pathfinder Mines)

Gas Hills, Wyoming (Union Carbide)

Powder River, Wyoming (Rocky Mountain Energy)

Powder River, Wyoming (Exxon Minerals)

Red Desert, Wyoming

Shirley Basin, Wyoming (Pathfinder Mines)

Shirley Basin, Wyoming (Petrotomics)

Shut-Down Mills (11)

Bluewater, New Mexico

Seboyeta, New Mexico

Church Rock, New Mexico

Marquez, New Mexico

Edgemont, South Dakota

Falls City, Texas (Conoco-Pioneer Nuclear)

Ray Point, Texas

Hanksville, Ut ah

Wellpinit, Washington

Jeffrey City, Wyoming

Gas Hills, Wyoming (Federal-American Partners)
Total

Population

349

5,933

396

1

453

8

343

2,361

411

0

0

0

6

0

0

357

Total

Population

907

166

312

15

1,421

42

130

171

49

906 
TABLE 6. Estimated Total Population Within 5 Kilometers of Inactive Uranium Mills

Total

Population

Monument Valley, Arizona

60

Tuba City, Arizona

Durango, Colorado

Grand Junction, Colorado

Gunnison, Colorado

Maybe 11 , Colorado

Naturita, Colorado (General Electric)

Naturita, Colorado (Ranchers Exploration)

New Rifle, Colorado

0ld Rifle, Colorado

Slick Rock, Colorado (North Continent)

Slick Rock, Colorado (Union Carbide)

Lowman, Idaho

Ambrosia Lake, New Mexico (Phillips Petroleum)

Shiprock, New Mexico

Belfield, North Dakota

Bowman, North Dakota

Lakeview, Oregon

Canonsburg, Pennsylvania

Falls City, Texas (Susquehanna Western)

Green River, Utah

Mexican Hat, Utah

Salt Lake City, Utah

Converse County, Wyoming

Riverton, Wyoming
64

12,058

38,011

7,315

0

896

723

5,659

10

4,184

22,135

45

1,498

303

91,498

18

11,738 


\section{REFERENCES}

1. Ford, Bacon \& Davis Utah, Inc. April 1981. Engineering Assessment of Inactive Uranium Mill Tailings - Vitro Site, Salt Lake City Utah. DOE/UMT-0102, prepared for the U.S. Department of Energy by Ford, Bacon \& Davis Utah, Inc., Salt Lake City, Utah.

2. Ford, Bacon \& Davis Utah, Inc. June 1981. Engineering Assessment of Inactive Uranium Mill Tailings - Durango Site, Durango, Colorado. D0E/UMT-0103, prepared for the U.S. Department of Energy by Ford, Bacon \& Davis Utah, Inc., Salt Lake City, Utah.

3. Ford, Bacon \& Davis Utah, Inc. July 1981. Engineering Assessment of Inactive Uranium Mill Tailings - Grand Junction Site, Grand Junction, Colorado. D0E/UMT-0105, prepared for the U.S. Department of Energy by Ford, Bacon \& Davis Utah, Inc., Salt Lake City, Utah.

4. Ford, Bacon \& Davis Utah, Inc. July 1981. Engineering Assessment of Inactive Uranium Mill Tailings - Naturita Site, Naturita, Colorado. DOE/UMT-0112, prepared for the U.S. Department of Energy by Ford, Bacon \& Davis Utah, Inc., Salt Lake City, Utah.

5. Ford, Bacon \& Davis Utah, Inc. July 1981. Engineering Assessment of Inactive Uranium Mill Tailings - Shiprock Site, Shiprock. New Mexico. DOE/UMT-0104, prepared for the U.S. Department of Energy by Ford, Bacon \& Davis Utah, Inc., Salt Lake City, Utah.

6. Ford, Bacon \& Davis Utah, Inc. August 1981. Engineering Assessment of Inactive Uranium Mill Tailings - New and 0ld Rifle Sites, Rifle, Colorado. DOE/UMT-0108, prepared for the U.S. Department of Energy by Ford, Bacon \& Davis Utah, Inc., Salt Lake City, Utah.

7. Ford, Bacon \& Davis Utah, Inc. August 1981. Engineering Assessment of Inactive Uranium Mill Tailings - Riverton Site, Riverton, Wyoming. DOE/UMT-0106, prepared for the U.S. Department of Energy by Ford, Bacon \& Davis Utah, Inc., Salt Lake City, Utah.

8. Ford, Bacon \& Davis Utah, Inc. September 1981. Engineering Assessment of Inactive Uranium Mill Tailings - Gunnison Site, Gunnison, CoTorado. D0E/UMT-01.07, prepared for the U.S. Department of Energy by Ford, Bacon \& Davis Utah, Inc., Salt Lake City, Utah.

9. Ford, Bacon \& Davis Utah, Inc. September 1981. Engineering Assessment of Inactive Uranium Mill Tailings - Lowman Site, Lowman, Idaho. DOE/UMT0118, prepared for the U.S. Department of Energy by Ford, Bacon \& Davis Utah, Inc., Salt Lake City, Utah.

10. Ford, Bacon \& Davis Utah, Inc. September 1981. Engineering Assessment of Inactive Uranium Mi 11 Tailings - Maybell Site, Maybe11, Colorado. D0E/UMT-0116, prepared for the U.S. Department of Energy by Ford, Bacon \& Davis Utah, Inc., Salt Lake City, Utah. 
11. Ford, Bacon \& Davis Utah, Inc. September 1981. Engineering Assessment of Inactive Uranium Mill Tailings - Mexican Hat Site, Mexican Hat, Utah. DOE/ UMT-0109, prepared for the U.S. Department of Energy by Ford, Bacon \& Davis Utah, Inc., Salt Lake City, Utah.

12. Ford, Bacon \& Davis Utah, Inc. September 1981. Engineering Assessment of Inactive Uranium Mill Tailings - Slick Rock Sites, Slick Rock, Colorado. DOE/UMT-0115, prepared for the U.S. Department of Energy by Ford, Bacon \& Davis Utah, Inc., Salt Lake City, Utah.

13. Ford, Bacon \& Davis Utah, Inc. September 1981. Engineering Assessment of Inactive Uranium Mill Tailings - Tuba City Site, Tuba City, Arizona. DOE/UMT-0120, prepared for the U.S. Department of Energy by Ford, Bacon \& Davis Utah, Inc., Salt Lake City, Utah.

14. Ford, Bacon \& Davis Utah, Inc. October 1981. Engineering Assessment of Inactive Uranium Mill Tailings - Falls City Site, Falls City, Texas. DOE/UMT-0111, prepared for the U.S. Department of Energy by Ford, Bacon \& Davis Utah, Inc., Salt Lake City, Utah.

15. Ford, Bacon \& Davis Utah, Inc. October 1981. Engineering Assessment of Inactive Uranium Mill Tailings - Lakeview Site, Lakeview, Oregon. DOE/UMT-0110, prepared for the U.S. Department of Energy by Ford, Bacon \& Davis Utah, Inc., Salt Lake City, Utah.

16. Ford, Bacon \& Davis Utah, Inc. October 1981. Engineering Assessment of Inactive Uranium Mill Tailings - Monument Valley Site, Monument Valley, Arizona. D0E/UMT-0117, prepared for the U.S. Department of Energy by Ford, Bacon \& Davis Utah, Inc., Salt Lake City, Utah.

17. Ford, Bacon \& Davis Utah, Inc. October 1981. Engineering Assessment of Inactive Uranium Mill Tailings - Philips/United Nuclear Site, Ambrosia Lake, New Mexico. DOE/UMT-0113, prepared for the U.S. Department of Energy by Ford, Bacon \& Davis Utah, Inc., Salt Lake City, Utah.

18. Ford, Bacon \& Davis Utah, Inc. October 1981. Engineering Assessment of Inactive Uranium Mill Tailings - Spook Site, Converse County, Wyoming. DOE/UMT-0119, prepared for the U.S. Department of Energy by Ford, Bacon \& Davis Utah, Inc., Salt Lake City, Utah.

19. Ford, Bacon \& Davis Utah, Inc. November 1981. Engineering Assessment of Inactive Uranium Mill Tailings - Belfield Site, Belfield, South Dakota. DOE/UMT-0122, prepared for the U.S. Department of Energy by Ford, Bacon \& Davis Utah, Inc., Salt Lake City, Utah.

20. Ford, Bacon \& Davis Utah, Inc. November 1981. Engineering Assessment of Inactive Uranium Mill Tailings - Bowman Site, Bowman, South Dakota. DOE/UMT-0121, prepared for the U.S. Department of Energy by Ford, Bacon \& Davis Utah, Inc., Salt Lake City, Utah. 
21. Douglas, Richard L., and Joseph M. Hans, Jr. August 1975. Gamma Radiation Surveys at Inactive Uranium Mill Sites. ORP/LV-75-5, prepared for the U.S. Environmental Protection Agency, Office of Radiation Programs - Las Vegas Facility, Las Vegas, Nevada.

22. Young, J.K., L.W. Long and J.W. Reis. April 1982. Environmental Factors Affecting Long-Term Stabilization of Radon Suppression Covers for Uranium Mill Tailings. NUREG/CR-2564, prepared for the U.S. Nuclear Regulatory Commission by Pacific Northwest Laboratory, Richland, Washington. 
APPENDIX A

MILL SITE POPULATION TABLES 

Latitude $38^{\circ} 22^{\prime} 00^{\prime \prime} \mathrm{N} \quad$ Longitude $108^{\circ} 45^{\prime} 00^{\prime \prime} \mathrm{W}$

Estimated Population - June 1983

Radial Distance $(\mathrm{km})$

\begin{tabular}{|c|c|c|c|c|c|c|}
\hline Direction & $0.0-0.5$ & $0.5-1.0$ & $1.0-2.0$ & $2.0-3.0$ & $3.0-4.0$ & $4.0-5.0$ \\
\hline$N$ & 0 & 45 & 108 & 0 & 0 & 0 \\
\hline NNE & 0 & 77 & 9 & 0 & 0 & 0 \\
\hline NE & 0 & 0 & 0 & 0 & 0 & 0 \\
\hline ENE & 0 & 11 & 0 & 0 & 0 & 0 \\
\hline$E$ & 0 & 14 & 28 & 0 & 0 & 0 \\
\hline ESE & 0 & 0 & 0 & 6 & 3 & 0 \\
\hline SE & 0 & 0 & 0 & 0 & 0 & 0 \\
\hline SSE & 0 & 0 & 0 & 0 & 0 & 0 \\
\hline$S$ & 0 & 0 & 0 & 0 & 0 & 0 \\
\hline SSW & 0 & 0 & 0 & 0 & 0 & 0 \\
\hline SW & 0 & 0 & 0 & 0 & 0 & 0 \\
\hline WSW & 0 & 0 & 0 & 0 & 0 & 0 \\
\hline$w$ & 0 & 0 & 0 & 0 & 0 & 0 \\
\hline WNW & 0 & 0 & 0 & 0 & 0 & 0 \\
\hline $\mathrm{NW}$ & 0 & 0 & 0 & 0 & 0 & 0 \\
\hline NNW & 0 & 0 & 48 & 0 & 0 & 0 \\
\hline Tota 1 & 0 & 147 & 193 & 6 & 3 & 0 \\
\hline
\end{tabular}

Total Population $(0.0-5.0 \mathrm{~km}) 349$

A. 1 
Site Canon City, Colorado

Latitude $38^{\circ} 23^{\prime} 46^{\prime \prime} \mathrm{N} \quad$ Longitude $105^{\circ} 13^{\prime} 45^{\prime \prime} \mathrm{W}$

Estimated Population - June 1983

\begin{tabular}{|c|c|c|c|c|c|c|}
\hline \multirow[b]{2}{*}{ Direction } & \multicolumn{5}{|c|}{ Radial Distance $(\mathrm{km})$} & \multirow[b]{2}{*}{$4.0-5.0$} \\
\hline & $\underline{0.0-0.5}$ & $0.5-1.0$ & $1.0-2.0$ & $2.0-3.0$ & $3.0-4.0$ & \\
\hline N & 0 & 0 & 0 & 5 & 935 & 1158 \\
\hline NNE & 0 & 0 & 0 & 124 & 899 & 715 \\
\hline NE & 0 & 0 & 0 & 39 & 715 & 303 \\
\hline ENE & 0 & 0 & 0 & 0 & 88 & 52 \\
\hline$E$ & 0 & 0 & 0 & 0 & 0 & 0 \\
\hline ESE & 0 & 0 & 0 & 0 & 0 & 0 \\
\hline SE & 0 & 0 & 0 & 0 & 0 & 0 \\
\hline SSE & 0 & 0 & 0 & 0 & 5 & 0 \\
\hline S & 0 & 0 & 0 & 0 & 3 & 8 \\
\hline SSW & 0 & 0 & 0 & 0 & 0 & 0 \\
\hline SW & 0 & 0 & 0 & 0 & 0 & 0 \\
\hline WSW & 0 & 0 & 0 & 16 & 0 & 0 \\
\hline W & 0 & 0 & 0 & 0 & 0 & 0 \\
\hline WNW & 0 & 0 & 0 & 0 & 0 & 0 \\
\hline NW & 0 & 0 & 0 & 0 & 0 & 47 \\
\hline NNW & 0 & 0 & 0 & 0 & 122 & 699 \\
\hline Tota 1 & 0 & 0 & 0 & 184 & 2,767 & 2,982 \\
\hline
\end{tabular}

Total Population $(0.0-5.0 \mathrm{~km}) 5933$ 
Site Milan, New Mexico

Latitude $35^{\circ} 14^{\prime} 31^{\prime \prime} \mathrm{N} \quad$ Longitude $107^{\circ} 51^{\prime} 46^{\prime \prime} \mathrm{W}$

Estimated Population - June 1983

\begin{tabular}{|c|c|c|c|c|c|c|}
\hline \multirow[b]{2}{*}{ Direction } & \multicolumn{6}{|c|}{ Radial Distance $(\mathrm{km})$} \\
\hline & $0.0-0.5$ & $\underline{0.5-1.0}$ & $1.0-2.0$ & $2.0-3.0$ & $3.0-4.0$ & $4.0-5.0$ \\
\hline$N$ & 0 & 0 & 0 & 0 & 0 & 0 \\
\hline NNE & 0 & 0 & 0 & 0 & 0 & 0 \\
\hline NE & 0 & 0 & 0 & 0 & 3 & 0 \\
\hline ENE & 0 & 0 & 0 & 0 & 0 & 0 \\
\hline E & 0 & 0 & 3 & 0 & 0 & 0 \\
\hline ESE & 0 & 0 & 0 & 0 & 0 & 0 \\
\hline SE & 0 & 0 & 0 & 0 & 0 & 0 \\
\hline SSE & 0 & 0 & 3 & 0 & 0 & 0 \\
\hline$S$ & 0 & 0 & 51 & 22 & 0 & 0 \\
\hline SSW & 0 & 0 & 67 & 16 & 29 & 41 \\
\hline SW & 0 & 0 & 44 & 3 & 0 & 10 \\
\hline WSW & 0 & 0 & 22 & 63 & 13 & 6 \\
\hline$W$ & 0 & 0 & 0 & 0 & 0 & 0 \\
\hline WNW & 0 & 0 & 0 & 0 & 0 & 0 \\
\hline$N W$ & 0 & 0 & 0 & 0 & 0 & 0 \\
\hline NNW & 0 & 0 & 0 & 0 & 0 & 0 \\
\hline Total & 0 & 0 & 190 & 104 & 45 & 57 \\
\hline
\end{tabular}

Total Population $(0.0-5.0 \mathrm{~km}) 396$

A. 3 
Site Ambrosia Lake, New Mexico (Kerr-McGee Nuclear)

Latitude $35^{\circ} 23^{\prime} 39^{\prime \prime} \mathrm{N} \quad$ Longitude $107^{\circ} 49^{\prime} 47^{\prime \prime} \mathrm{W}$

Estimated Population - June 1983

Radial Distance $(\mathrm{km})$

\begin{tabular}{|c|c|c|c|c|c|c|}
\hline Direction & $0.0-0.5$ & $0.5-1.0$ & $1.0-2.0$ & $2.0-3.0$ & $3.0-4.0$ & $4.0-5.0$ \\
\hline N & 0 & 0 & 0 & 0 & 0 & 0 \\
\hline NNE & 0 & 0 & 0 & 0 & * & * \\
\hline NE & 0 & 0 & 0 & * & * & * \\
\hline ENE & 0 & 0 & 0 & * & * & * \\
\hline$E$ & 0 & 0 & 0 & 0 & * & * \\
\hline ESE & 0 & 0 & 0 & 0 & 0 & $\star$ \\
\hline SE & 0 & 0 & 0 & 0 & 0 & 0 \\
\hline SSE & 0 & 0 & 0 & 0 & 0 & 0 \\
\hline S & 0 & 0 & 0 & 0 & 0 & 0 \\
\hline SSW & 0 & 0 & 0 & 0 & 0 & 0 \\
\hline SW & 0 & 0 & 0 & 0 & 0 & 0 \\
\hline WSW & 0 & 0 & 0 & 0 & 0 & 0 \\
\hline W & 0 & 0 & 0 & 0 & 0 & 0 \\
\hline WNW & 0 & 0 & 0 & 0 & 0 & 0 \\
\hline NW & 0 & 0 & 0 & 0 & 0 & 0 \\
\hline NNW & 0 & 0 & 0 & 1 & 0 & 0 \\
\hline Total & 0 & 0 & 0 & 1 & 0 & 0 \\
\hline
\end{tabular}

Total Population $(0.0-5.0 \mathrm{~km}) .1$

* Sector lies within area apportioned to Ambrosia Lake, New Mexico (Phillips Petroleum) mill.

A. 4 
Site Panna Maria, Texas

Latitude $28^{\circ} 57^{\prime} 33^{\prime \prime} \mathrm{N} \quad$ Longitude $97^{\circ} 56^{\prime} 31^{\prime \prime} \mathrm{W}$

Estimated Population - June 1983

Radial Distance (km)

$\begin{array}{lcccccc}\text { Direction } & \frac{0.0-0.5}{0.5-1.0} & \frac{1.0-2.0}{2.0-3.0} & \frac{3.0-4.0}{4.0-5.0} \\ \text { N } & 0 & 0 & 0 & 0 & 0 & 0 \\ \text { NNE } & 0 & 0 & 3 & 0 & 0 & 9 \\ \text { NE } & 0 & 0 & 3 & 0 & 6 & 3 \\ \text { ENE } & 0 & 0 & 0 & 6 & 0 & 18 \\ \text { E } & 0 & 0 & 9 & 12 & 21 & 68 \\ \text { ESE } & 0 & 0 & 9 & 3 & 9 & 0 \\ \text { SE } & 0 & 0 & 6 & 0 & 0 & 3 \\ \text { SSE } & 0 & 6 & 0 & 0 & 0 & 0 \\ \text { S } & 0 & 6 & 9 & 0 & 0 & 6 \\ \text { SSW } & 0 & 0 & 3 & 0 & 6 & 24 \\ \text { SW } & 0 & 0 & 0 & 0 & 3 & 6 \\ \text { WSW } & 0 & 0 & 0 & 6 & 12 & 9 \\ \text { W } & 0 & 0 & 0 & 3 & 15 & 136 \\ \text { WNW } & 0 & 0 & 0 & 0 & 6 & 0 \\ \text { NW } & 0 & 0 & 0 & 3 & 3 & 0 \\ \text { NNW } & 0 & 0 & 0 & 0 & 0 & 3 \\ \text { Total } & 0 & 12 & 42 & 33 & 81 & 285\end{array}$

Total Population $(0.0-5.0 \mathrm{~km}) 453$

A. 5 
Latitude $37^{\circ} 31^{\prime} 37^{\prime \prime} \mathrm{N} \quad$ Longitude $109^{\circ} 30^{\prime} 33^{\prime \prime} \mathrm{W}$

Estimated Population - June 1983

Radial Distance (km)

\begin{tabular}{|c|c|c|c|c|c|c|}
\hline Direction & $0.0-0.5$ & $0.5-1.0$ & $1.0-2.0$ & $2.0-3.0$ & $3.0-4.0$ & $4.0-5.0$ \\
\hline$N$ & 0 & 0 & 0 & 0 & 0 & 0 \\
\hline NNE & 0 & 0 & 0 & 0 & 0 & 4 \\
\hline NE & 0 & 0 & 0 & 0 & 0 & 0 \\
\hline ENE & 0 & 0 & 0 & 0 & 0 & 0 \\
\hline$E$ & 0 & 0 & 0 & 0 & 0 & 0 \\
\hline ESE & 0 & 0 & 0 & 0 & 0 & 0 \\
\hline SE & 0 & 0 & 0 & 0 & 0 & 0 \\
\hline SSE & 0 & 0 & 0 & 0 & 0 & 4 \\
\hline$S$ & 0 & 0 & 0 & 0 & 0 & 0 \\
\hline SSW & 0 & 0 & 0 & 0 & 0 & 0 \\
\hline SW & 0 & 0 & 0 & 0 & 0 & 0 \\
\hline WSW & 0 & 0 & 0 & 0 & 0 & 0 \\
\hline W & 0 & 0 & 0 & 0 & 0 & 0 \\
\hline WNW & 0. & 0 & 0 & 0 & 0 & 0 \\
\hline NW & 0 & 0 & 0 & 0 & 0 & 0 \\
\hline NNW & 0 & 0 & 0 & 0 & 0 & 0 \\
\hline Total & 0 & 0 & 0 & 0 & 0 & 8 \\
\hline
\end{tabular}

Total Population $(0.0-5.0 \mathrm{~km}) 8$ 
Site La Sal, Utah

Latitude $38^{\circ} 16^{\prime} 00^{\prime \prime}$ Longitude $109^{\circ} 16^{\prime} 30^{\prime \prime} \mathrm{W}$

Estimated Population - June 1983

Radial Distance $(\mathrm{km})$

Direction $\quad \underline{0.0-0.5} \quad \underline{0.5-1.0} \quad \underline{1.0-2.0} \quad \underline{2.0-3.0} \quad \underline{3.0-4.0} \quad \underline{4.0-5.0}$

N

0

53

85

12

0

NNE

0

8

28

57

0

40

NE

0

0

4

0

0

ENE

0

0

0

0

0

E

0

0

0

0

0

0

ESE

0

0

0

0

SE

SSE

$\mathrm{S}$

SSW

SW

WSW

0

0

0

0

0

0

0

0

0

0

0

0

0

0

W

WNW

NW

NNW

Total

0

8

105

154

32

44

Total Population $(0.0-5.0 \mathrm{~km}) 343$ 
Latitude $38^{\circ} 35^{\prime} 59^{\prime \prime} \mathrm{N} \quad$ Longitude $109^{\circ} 35^{\prime} 44^{\prime \prime} \mathrm{W}$

Estimated Population - June 1983

\begin{tabular}{|c|c|c|c|c|c|c|}
\hline \multirow[b]{2}{*}{ Direction } & \multicolumn{6}{|c|}{ Radial Distance $(\mathrm{km})$} \\
\hline & $0.0-0.5$ & $0.5-1.0$ & $1.0-2.0$ & $2.0-3.0$ & $3.0-4.0$ & $4.0-5.0$ \\
\hline N & 0 & 0 & 0 & 0 & 0 & 0 \\
\hline NNE & 0 & 0 & 0 & 0 & 0 & 0 \\
\hline$N E$ & 0 & 0 & 0 & 0 & 0 & 0 \\
\hline ENE & 0 & 0 & 6 & 0 & 0 & 0 \\
\hline$E$ & 0 & 0 & 3 & 3 & 0 & 0 \\
\hline ESE & 0 & 0 & 0 & 9 & 674 & 668 \\
\hline SE & 0 & 0 & 0 & 0 & 420 & 557 \\
\hline SSE & 0 & 0 & 0 & 0 & 0 & 0 \\
\hline S & 0 & 0 & 0 & 0 & 0 & 0 \\
\hline SSW & 0 & 0 & 0 & 0 & 0 & 0 \\
\hline SW & 0 & 0 & 0 & 0 & 0 & 0 \\
\hline WSW & 0 & 0 & 0 & 0 & 0 & 0 \\
\hline W & 0 & 0 & 0 & 0 & 0 & 0 \\
\hline WNW & 0 & 0 & 0 & 0 & 0 & 0 \\
\hline NW & 0 & 0 & 0 & 21 & 0 & 0 \\
\hline NNW & 0 & 0 & 0 & 0 & 0 & 0 \\
\hline Total & 0 & 0 & 9 & 33 & 1094 & 1225 \\
\hline
\end{tabular}

Total Population $(0.0-5.0 \mathrm{~km}) 2361$ 
Latitude $47^{\circ} 54^{\prime} 06^{\prime \prime} \mathrm{N} \quad$ Longitude $117^{\circ} 49^{\prime} 58^{\prime \prime} \mathrm{W}$

Estimated Population - June 1983

\begin{tabular}{|c|c|c|c|c|c|c|}
\hline \multirow[b]{2}{*}{ Direction } & \multicolumn{5}{|c|}{ Radial Distance $(\mathrm{km})$} & \multirow[b]{2}{*}{$\underline{4.0-5.0}$} \\
\hline & $0.0-0.5$ & $0.5-1.0$ & $1.0-2.0$ & $2.0-3.0$ & $3.0-4.0$ & \\
\hline N & 0 & 0 & 20 & 12 & 15 & 0 \\
\hline NNE & 0 & 0 & 20 & 3 & 6 & 0 \\
\hline $\mathrm{NE}$ & 0 & 0 & 15 & 12 & 20 & 12 \\
\hline ENE & 0 & 0 & 29 & 29 & 6 & 0 \\
\hline E & 0 & 3 & 0 & 0 & 0 & 0 \\
\hline ESE & 0 & 0 & 0 & 0 & 0 & 3 \\
\hline SE & 0 & 0 & 0 & 0 & 3 & 35 \\
\hline SSE & 0 & 0 & 0 & 0 & 0 & 0 \\
\hline$S$ & 0 & 0 & 0 & 3 & 3 & 3 \\
\hline SSW & 0 & 0 & 0 & 0 & 9 & 0 \\
\hline SW & 0 & 0 & 0 & 0 & 3 & 3 \\
\hline WSW & 0 & 0 & 0 & 3 & 15 & 0 \\
\hline$W$ & 0 & 0 & 0 & 58 & 6 & 3 \\
\hline WNW & 0 & 0 & 0 & 23 & 0 & 0 \\
\hline NW & 0 & 0 & 0 & 3 & 0 & 0 \\
\hline NNW & 0 & 0 & 9 & 12 & 0 & 0 \\
\hline Total & 0 & 3 & 93 & 157 & 96 & 62 \\
\hline
\end{tabular}

Total Population $(0.0-5.0 \mathrm{~km}) 411$ 
Site Gas Hills, Wyoming (Pathfinder Mines)

Latitude $42^{\circ} 49^{\prime} 55^{\prime \prime} \mathrm{N} \quad$ Longitude $107^{\circ} 29^{\prime} 23^{\prime \prime} \mathrm{W}$

Estimated Population - June 1983

Radial Distance $(\mathrm{km})$

\begin{tabular}{|c|c|c|c|c|c|c|}
\hline Direction & $0.0-0.5$ & $0.5-1.0$ & $1.0-2.0$ & $2.0-3.0$ & $3.0-4.0$ & $4.0-5.0$ \\
\hline N & 0 & 0 & 0 & 0 & 0 & 0 \\
\hline NNE & 0 & 0 & 0 & 0 & 0 & 0 \\
\hline NE & 0 & 0 & 0 & 0 & 0 & 0 \\
\hline ENE & 0 & 0 & 0 & 0 & 0 & 0 \\
\hline$E$ & 0 & 0 & 0 & 0 & 0 & 0 \\
\hline ESE & 0 & 0 & 0 & 0 & 0 & 0 \\
\hline SE & 0 & 0 & 0 & 0 & 0 & 0 \\
\hline SSE & 0 & 0 & 0 & 0 & 0 & * \\
\hline$S$ & 0 & 0 & 0 & 0 & * & * \\
\hline SSW & 0 & 0 & 0 & * & * & * \\
\hline SW & 0 & 0 & 0 & 0 & * & * \\
\hline WSW & 0 & 0 & 0 & 0 & 0 & * \\
\hline$W$ & 0 & 0 & 0 & 0 & 0 & 0 \\
\hline WNW & 0 & 0 & 0 & 0 & 0 & 0 \\
\hline NW & 0 & 0 & 0 & 0 & 0 & 0 \\
\hline NNW & 0 & 0 & 0 & 0 & 0 & 0 \\
\hline Total & 0 & 0 & 0 & 0 & 0 & 0 \\
\hline
\end{tabular}

Total Population $(0.0-5.0 \mathrm{~km})$ ?

* Sector lies within area apportioned to Gas Hills, Wyoming (Federal-American Partners) mill.

A. 10 
Site Gas Hills, Wyoming (Union Carbide)

Latitude $42^{\circ} 49^{\prime} 45^{\prime \prime} \mathrm{N} \quad$ Longitude $107^{\circ} 29^{\prime} 34^{\prime \prime} \mathrm{W}$

Estimated Population - June 1983

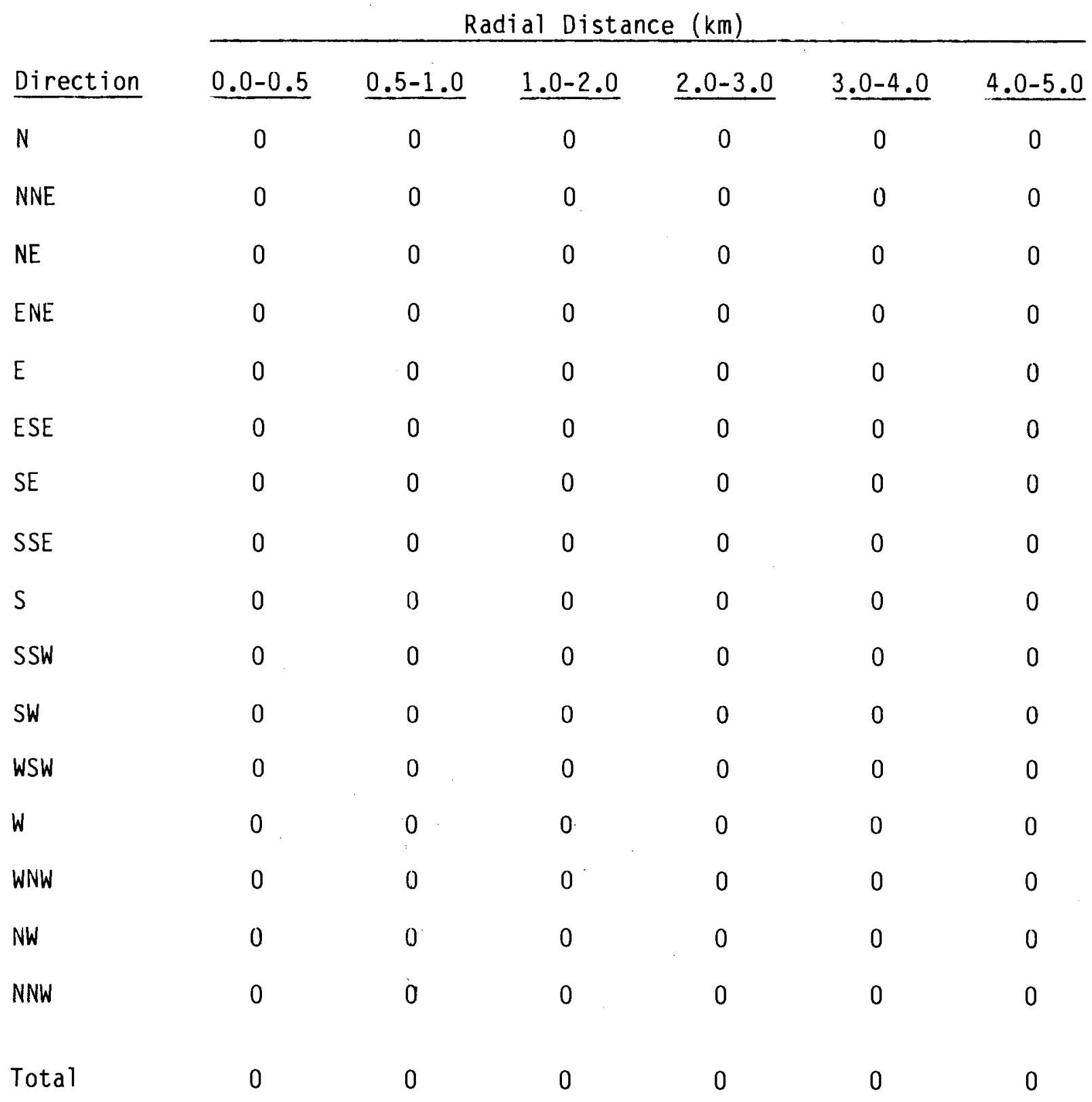

Total Population $(0.0-5.0 \mathrm{~km}) \underline{0}$

A. 11 
Site Powder River, Wyoming (Rocky Mountain Energy)

Latitude $43^{\circ} 16^{\prime} 11^{\prime \prime} \mathrm{N}$ Longitude $105^{\circ} 37^{\prime} 46^{\prime \prime} \mathrm{W}$

Estimated Population - June 1983

Radial Distance (km)

$\begin{array}{lcccccc}\text { Direction } & \underline{0.0-0.5} & \frac{0.5-1.0}{1.0-2.0} & \frac{2.0-3.0}{3.0-4.0} & \frac{4.0-5.0}{0} \\ \text { N } & 0 & 0 & 0 & 0 & 0 & 0 \\ \text { NNE } & 0 & 0 & 0 & 0 & 0 & 0 \\ \text { NE } & 0 & 0 & 0 & 0 & 0 & 0 \\ \text { ENE } & 0 & 0 & 0 & 0 & 0 & 0 \\ \text { E } & 0 & 0 & 0 & 0 & 0 & 0 \\ \text { ESE } & 0 & 0 & 0 & 0 & 0 & 0 \\ \text { SE } & 0 & 0 & 0 & 0 & \star & * \\ \text { SSE } & 0 & 0 & 0 & * & * & * \\ \text { S } & 0 & 0 & 0 & * & \star & * \\ \text { SSW } & 0 & 0 & 0 & 0 & \star & * \\ \text { SW } & 0 & 0 & 0 & 0 & 0 & 0 \\ \text { WSW } & 0 & 0 & 0 & 0 & 0 & 0 \\ \text { W } & 0 & 0 & 0 & 0 & 0 & 0 \\ \text { WNW } & 0 & 0 & 0 & 0 & 0 & 0 \\ \text { NW } & 0 & 0 & 0 & 0 & 0 & 0 \\ \text { NNW } & 0 & 0 & 0 & 0 & 0 & 0 \\ \text { Total } & 0 & 0 & 0 & 0 & 0 & 0\end{array}$

Total Population $(0.0-5.0 \mathrm{~km}) 0$

* Sector lies within area apportioned to Converse County, Wyoming mill.

A. 12 
Site Powder River, Wyoming (Exxon Minerals)

Latitude $43^{\circ} 04^{\prime} 07^{\prime \prime} \mathrm{N} \quad$ Longitude $105^{\circ} 29^{\prime} 49^{\prime \prime} \mathrm{W}$

Est imated Population - June 1983

\begin{tabular}{|c|c|c|c|c|c|c|c|}
\hline \multirow{2}{*}{ - } & \multirow[b]{2}{*}{ Direction } & \multicolumn{6}{|c|}{ Radial Distance $(\mathrm{km})$} \\
\hline & & $0.0-0.5$ & $0.5-1.0$ & $1.0-2.0$ & $2.0-3.0$ & $3.0-4.0$ & $4.0-5.0$ \\
\hline- & N & 0 & 0 & 0 & 0 & 6 & 0 \\
\hline & NNE & 0 & 0 & 0 & 0 & 0 & 0 \\
\hline- & $\mathrm{NE}$ & 0 & 0 & 0 & 0 & 0 & 0 \\
\hline$=x$ & ENE & 0 & 0 & 0 & 0 & 0 & 0 \\
\hline$\ldots$ & $E$ & 0 & 0 & 0 & 0 & 0 & 0 \\
\hline$m$ & ESE & 0 & 0 & 0 & 0 & 0 & 0 \\
\hline & SE & 0 & 0 & 0 & 0 & 0 & 0 \\
\hline- & SSE & 0 & 0 & 0 & 0 & 0 & 0 \\
\hline 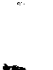 & $S$ & 0 & 0 & 0 & 0 & 0 & 0 \\
\hline .. & SSW & 0 & 0 & 0 & 0 & 0 & 0 \\
\hline- & SW & 0 & 0 & 0 & 0 & 0 & 0 \\
\hline$\ldots$ & WSW & 0 & 0 & 0 & 0 & 0 & 0 \\
\hline- & $w$ & 0 & 0 & 0 & 0 & 0 & 0 \\
\hline & WNW & 0 & 0 & 0 & 0 & 0 & 0 \\
\hline & NW & 0 & 0 & 0 & 0 & 0 & 0 \\
\hline 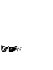 & NNW & 0 & 0 & 0 & 0 & 0 & 0 \\
\hline & Total & 0 & 0 & 0 & 0 & 6 & 0 \\
\hline & & & I Populat & $n(0.0-5$ & km) 6 & & \\
\hline & & & & A. 13 & & & \\
\hline
\end{tabular}


Site Red Desert, Wyoming

Latitude $42^{\circ} 02^{\prime} 56^{\prime \prime} \mathrm{N} \quad$ Longitude $107^{\circ} 53^{\prime} 28^{\prime \prime} \mathrm{W}$

Estimated Population - June 1983

Radial Distance $(\mathrm{km})$

\begin{tabular}{|c|c|c|c|c|c|c|}
\hline Direction & $\underline{0.0-0.5}$ & $0.5-1.0$ & $1.0-2.0$ & $2.0-3.0$ & $3.0-4.0$ & $4.0-5.0$ \\
\hline $\mathrm{N}$ & 0 & 0 & 0 & 0 & 0 & 0 \\
\hline NNE & 0 & 0 & 0 & 0 & 0 & 0 \\
\hline $\mathrm{NE}$ & 0 & 0 & 0 & 0 & 0 & 0 \\
\hline ENE & 0 & 0 & 0 & 0 & 0 & 0 \\
\hline$E$ & 0 & 0 & 0 & 0 & 0 & 0 \\
\hline ESE & 0 & 0 & 0 & 0 & 0 & 0 \\
\hline SE & 0 & 0 & 0 & 0 & 0 & 0 \\
\hline SSE & 0 & 0 & 0 & 0 & 0 & 0 \\
\hline $\mathrm{S}$ & 0 & 0 & 0 & 0 & 0 & 0 \\
\hline SSW & 0 & 0 & 0 & 0 & 0 & 0 \\
\hline SW & 0 & 0 & 0 & 0 & 0 & 0 \\
\hline WSW & 0 & 0 & 0 & 0 & 0 & 0 \\
\hline$W$ & 0 & 0 & 0 & 0 & 0 & 0 \\
\hline WNW & 0 & 0 & 0 & 0 & 0 & 0 \\
\hline $\mathrm{NW}$ & 0 & 0 & 0 & 0 & 0 & 0 \\
\hline NNW & 0 & 0 & 0 & 0 & 0 & 0 \\
\hline Total & 0 & 0 & 0 & 0 & 0 & 0 \\
\hline
\end{tabular}

Total Population $(0.0-5.0 \mathrm{~km}) 0$

A. 14 
Site Shirley Basin, Wyoming (Pathfinder Mines)

Latitude $42^{\circ} 22^{\prime} 14^{\prime \prime} \mathrm{N} \quad$ Longitude $106^{\circ} 10^{\prime} 37^{\prime \prime} \mathrm{W}$

\begin{tabular}{|c|c|c|c|c|c|c|}
\hline \multirow[b]{2}{*}{ Direction } & \multicolumn{5}{|c|}{ Estimated Population - June 1983} & \multirow[b]{2}{*}{$4.0-5.0$} \\
\hline & $\underline{0.0-0.5}$ & $0.5-1.0$ & $\underline{1.0-2.0}$ & $2.0-3.0$ & $3.0-4.0$ & \\
\hline $\mathrm{N}$ & 0 & 0 & 0 & 0 & 0 & 0 \\
\hline NNE & 0 & 0 & 0 & 0 & 0 & 0 \\
\hline $\mathrm{NE}$ & 0 & 0 & 0 & 0 & 0 & 0 \\
\hline ENE & 0 & 0 & 0 & 0 & 0 & 0 \\
\hline$E$ & 0 & 0 & 0 & 0 & 0 & 0 \\
\hline ESE & 0 & 0 & 0 & 0 & 0 & 0 \\
\hline SE & 0 & 0 & 0 & 0 & 0 & 0 \\
\hline SSE & 0 & 0 & 0 & 0 & 0 & * \\
\hline $\mathrm{s}$ & 0 & 0 & 0 & 0 & * & * \\
\hline SSW & 0 & 0 & 0 & 0 & * & * \\
\hline SW & 0 & 0 & 0 & 0 & * & * \\
\hline WSW & 0 & 0 & 0 & 0 & 0 & * \\
\hline$w$ & 0 & 0 & 0 & 0 & 0 & 0 \\
\hline WNW & 0 & 0 & 0 & 0 & 0 & 0 \\
\hline NW & 0 & 0 & 0 & 0 & 0 & 0 \\
\hline NNW & 0 & 0 & 0 & 0 & 0 & 0 \\
\hline Total & 0 & 0 & 0 & 0 & 0 & 0 \\
\hline
\end{tabular}

Total Population $(0.0-5.0 \mathrm{~km}) \underline{0}$

* Sector lies within area apportioned to Shirley Basin, Wyoming (Petrotomics) mill.

A. 15 
Site Shirley Basin, Wyoming (Petrotomics)

Latitude $42^{\circ} 20^{\prime} 04^{\prime \prime} \mathrm{N} \quad$ Longitude $106^{\circ} 11^{\prime} 49^{\prime \prime} \mathrm{W}$

Estimated Population - June 1983

Radial Distance $(\mathrm{km})$

\begin{tabular}{|c|c|c|c|c|c|c|}
\hline Direction & $0.0-0.5$ & $0.5-1.0$ & $1.0-2.0$ & $2.0-3.0$ & $3.0-4.0$ & $\underline{4.0-5.0}$ \\
\hline N & 0 & 0 & 0 & 0 & * & * \\
\hline NNE & 0 & 0 & 0 & 0 & * & * \\
\hline NE & 0 & 0 & 0 & 0 & * & * \\
\hline ENE & 0 & 0 & 0 & 0 & 0 & * \\
\hline E & 0 & 0 & 0 & 0 & 0 & 0 \\
\hline ESE & 0 & 0 & 0 & 0 & 0 & 0 \\
\hline SE & 0 & 0 & 0 & 0 & 0 & 0 \\
\hline SSE & 0 & 0 & 0 & 0 & 0 & 0 \\
\hline$S$ & 0 & 0 & 0 & 0 & 178 & 0 \\
\hline SSW & 0 & 0 & 0 & 0 & 179 & 0 \\
\hline SW & 0 & 0 & 0 & 0 & 0 & 0 \\
\hline WSW & 0 & 0 & 0 & 0 & 0 & 0 \\
\hline W & 0 & 0 & 0 & 0 & 0 & 0 \\
\hline WNW & 0 & 0 & 0 & 0 & 0 & 0 \\
\hline NW & 0 & 0 & 0 & 0 & 0 & 0 \\
\hline NNW & 0 & 0 & 0 & 0 & 0 & * \\
\hline Total & 0 & 0 & 0 & 0 & 357 & 0 \\
\hline
\end{tabular}

Total Population $(0.0-5.0 \mathrm{~km}) 357$

* Sector lies within area apportioned to Shirley Basin, Wyoming (Pathfinder Mines) mill. A.16 
Latitude $35^{\circ} 16^{\prime} 12^{\prime \prime} \mathrm{N} \quad$ Longitude $107^{\circ} 56^{\prime} 44^{\prime \prime} \mathrm{W}$

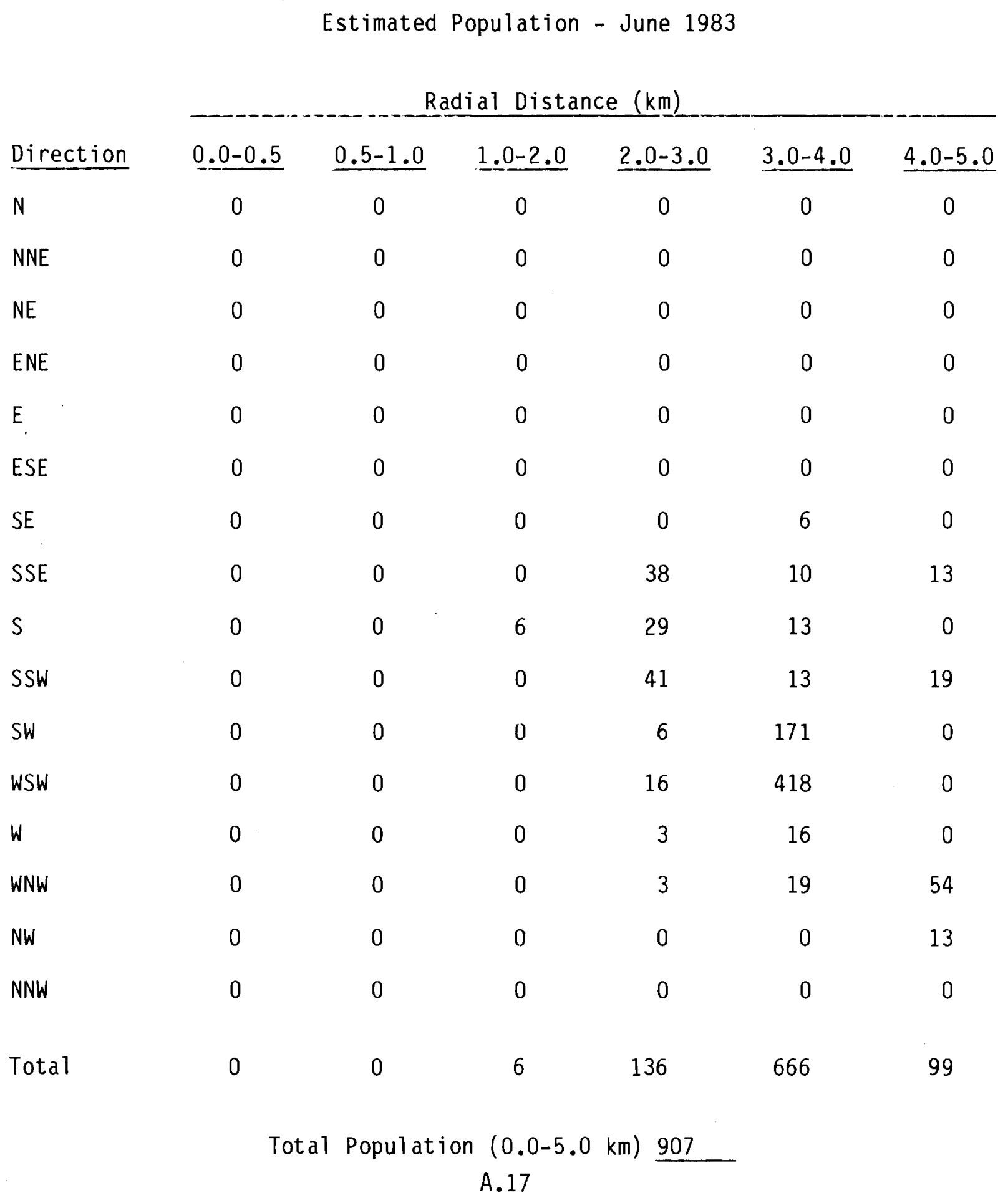


Site Seboyeta, New Mexico

Latitude $35^{\circ} 11^{\prime} 09^{\prime \prime} \mathrm{N} \quad$ Longitude $107^{\circ} 20^{\prime} 09^{\prime \prime} \mathrm{W}$

Estimated Population - June 1983

\begin{tabular}{|c|c|c|c|c|c|c|}
\hline \multirow[b]{2}{*}{ Direction } & \multicolumn{5}{|c|}{ Radial Distance (km) } & \multirow[b]{2}{*}{$4.0-5.0$} \\
\hline & $0.0-0.5$ & $0.5-1.0$ & $1.0-2.0$ & $2.0-3.0$ & $3.0-4.0$ & \\
\hline$N$ & 0 & 0 & 0 & 0 & 0 & 0 \\
\hline NNE & 0 & 0 & 0 & 0 & 0 & 0 \\
\hline NE & 0 & 0 & 0 & 0 & 0 & 0 \\
\hline ENE & 0 & 0 & 0 & 0 & 0 & 0 \\
\hline$E$ & 0 & 0 & 0 & 0 & 0 & 0 \\
\hline ESE & 0 & 0 & 0 & 0 & 0 & 0 \\
\hline SE & 0 & 0 & 0 & 0 & 0 & 0 \\
\hline SSE & 0 & 0 & 0 & 0 & 0 & 0 \\
\hline$S$ & 0 & 0 & 0 & 0 & 0 & 0 \\
\hline SSW & 0 & 0 & 0 & 0 & 0 & 0 \\
\hline SW & 0 & 0 & 0 & 0 & 0 & 0 \\
\hline WSW & 0 & 0 & 0 & 0 & 32 & 19 \\
\hline W & 0 & 0 & 0 & 0 & 0 & 35 \\
\hline WNW & 0 & 0 & 0 & 0 & 10 & 70 \\
\hline NW & 0 & 0 & 0 & 0 & 0 & 0 \\
\hline NNW & 0 & 0 & 0 & 0 & 0 & 0 \\
\hline Total & 0 & 0 & 0 & 0 & 42 & 124 \\
\hline
\end{tabular}

Total Population $(0.0-5.0 \mathrm{~km}) \underline{166}$ 
Latitude $35^{\circ} 38^{\prime} 47^{\prime \prime} \mathrm{N} \quad$ Longitude $108^{\circ} 30^{\prime} 08^{\prime \prime} \mathrm{W}$

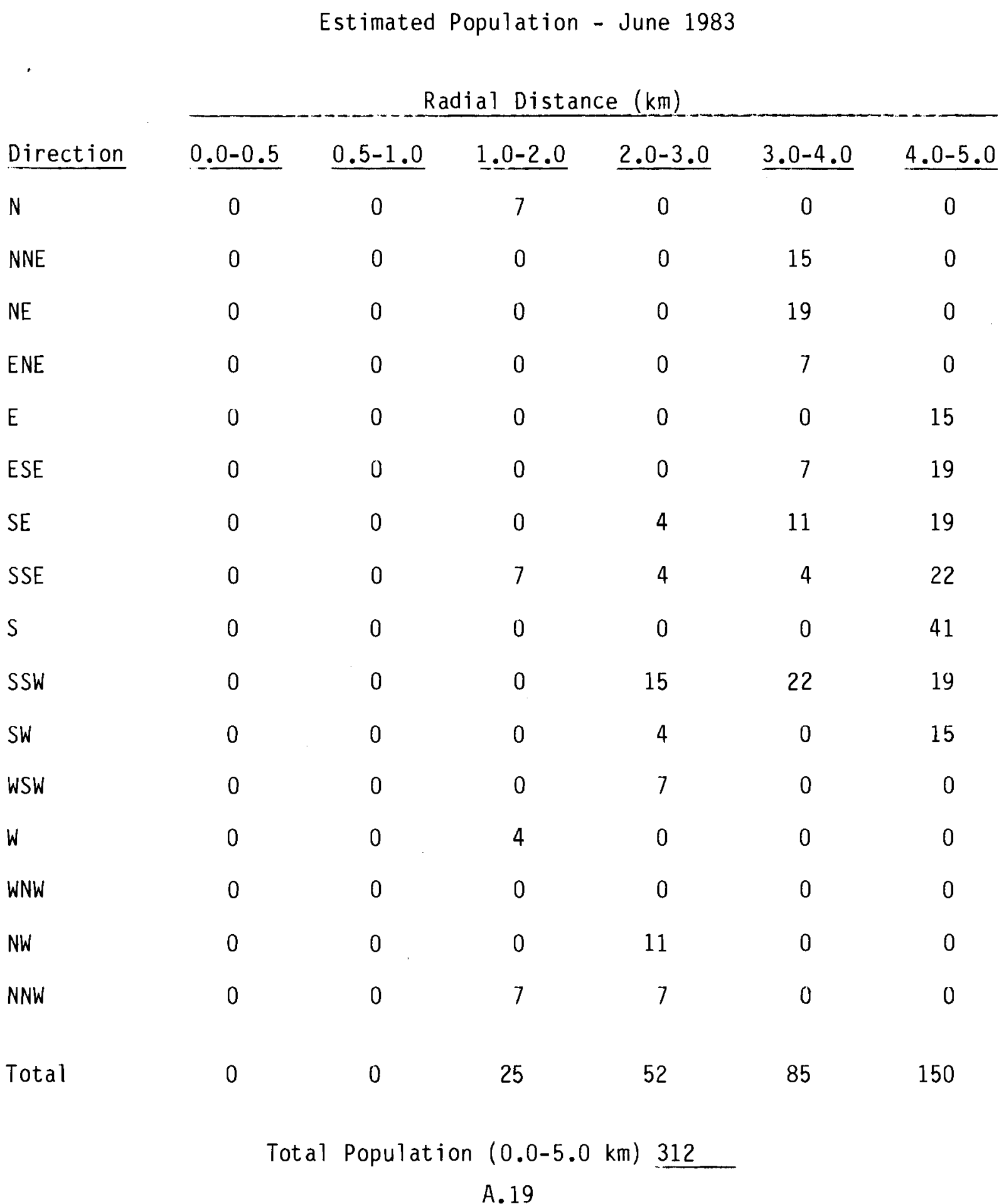


Site Marquez, New Mexico

Latitude $35^{\circ} 18^{\prime} 59^{\prime \prime} \mathrm{N}$ Longitude $107^{\circ} 16^{\prime} 28^{\prime \prime} \mathrm{W}$

Estimated Population - June 1983

Radial Distance $(\mathrm{km})$

\begin{tabular}{|c|c|c|c|c|c|c|}
\hline Direction & $0.0-0.5$ & $0.5-1.0$ & $1.0-2.0$ & $2.0-3.0$ & $3.0-4.0$ & $4.0-5.0$ \\
\hline N & 0 & 0 & 0 & 0 & 0 & 0 \\
\hline NNE & 0 & 0 & 0 & 0 & 0 & 0 \\
\hline$N E$ & 0 & 0 & 0 & 0 & 0 & 0 \\
\hline ENE & 0 & 0 & 0 & 0 & 0 & 0 \\
\hline$E$ & 0 & 0 & 0 & 0 & 0 & 0 \\
\hline ESE & 0 & 0 & 0 & 0 & 0 & 0 \\
\hline SE & 0 & 0 & 0 & 0 & 0 & 0 \\
\hline SSE & 0 & 0 & 0 & 0 & 0 & 0 \\
\hline$S$ & 0 & 0 & 0 & 0 & 0 & 0 \\
\hline SSW & 0 & 0 & 0 & 0 & 0 & 0 \\
\hline SW & 0 & 0 & 0 & 0 & 0 & 0 \\
\hline WSW & 0 & 0 & 0 & 0 & 0 & 0 \\
\hline W & 0 & 0 & 0 & 15 & 0 & 0 \\
\hline WNW & 0 & 0 & 0 & 0 & 0 & 0 \\
\hline NW & 0 & 0 & 0 & 0 & 0 & 0 \\
\hline NNW & 0 & 0 & 0 & 0 & 0 & 0 \\
\hline Total & 0 & 0 & 0 & 15 & 0 & 0 \\
\hline
\end{tabular}

Total Population $(0.0-5.0 \mathrm{~km}) 15$

A.20 
Site Edgemont, South Dakota

Latitude $43^{\circ} 17^{\prime} 43^{\prime \prime} \mathrm{N} \quad$ Longitude $103^{\circ} 48^{\prime} 46^{\prime \prime} \mathrm{W}$

Estimated Population - June 1983

\section{Radial Distance $(\mathrm{km})$}

\begin{tabular}{|c|c|c|c|c|c|c|}
\hline Direction & $0.0-0.5$ & $0.5-1.0$ & $1.0-2.0$ & $2.0-3.0$ & $3.0-4.0$ & $4.0-5.0$ \\
\hline$N$ & 0 & 0 & 5 & 8 & 0 & 0 \\
\hline NNE & 0 & 0 & 3 & 5 & 8 & 10 \\
\hline NE & 0 & 0 & 0 & 3 & 0 & 0 \\
\hline ENE & 0 & 0 & 0 & 0 & 0 & 0 \\
\hline$E$ & 0 & 0 & 0 & 0 & 0 & 0 \\
\hline ESE & 0 & 0 & 0 & 3 & 3 & 0 \\
\hline SE & 0 & 0 & 0 & 0 & 3 & 0 \\
\hline SSE & 0 & 0 & 0 & 0 & 3 & 0 \\
\hline S & 0 & 0 & 0 & 0 & 0 & 3 \\
\hline SSW & 0 & 0 & 0 & 0 & 0 & 0 \\
\hline SW & 0 & 0 & 20 & 0 & 3 & 5 \\
\hline WSW & 3 & 0 & 63 & 0 & 0 & 0 \\
\hline$W$ & 18 & 20 & 306 & 58 & 0 & 0 \\
\hline WNW & 10 & 18 & 612 & 94 & 8 & 0 \\
\hline $\mathrm{NW}$ & 0 & 0 & 83 & 0 & 0 & 10 \\
\hline NNW & 0 & 0 & 33 & 0 & 0 & 0 \\
\hline Total & 31 & 38 & 1,125 & 171 & 28 & 28 \\
\hline
\end{tabular}

Total Population $(0.0-5.0 \mathrm{~km}) 1,421$

A. 21 
Site Falls City, Texas (Conoco - Pioneer Nuclear)

Latitude $28^{\circ} 54^{\prime} 03^{\prime \prime} \mathrm{N} \quad$ Longitude $98^{\circ} 05^{\prime} 40^{\prime \prime} \mathrm{W}$

Estimated Population - June 1983

Radial Distance (km)

\begin{tabular}{|c|c|c|c|c|c|c|}
\hline Direction & $0.0-0.5$ & $\underline{0.5-1.0}$ & $1.0-2.0$ & $2.0-3.0$ & $3.0-4.0$ & $4.0-5.0$ \\
\hline$N$ & 0 & 0 & 0 & 0 & 0 & 0 \\
\hline NNE & 0 & 0 & 0 & 3 & 0 & 3 \\
\hline NE & 0 & 0 & 0 & 3 & 6 & 0 \\
\hline ENE & 0 & 0 & 0 & 0 & 0 & 0 \\
\hline$E$ & 0 & 0 & 0 & 0 & 0 & 0 \\
\hline ESE & 0 & 0 & 0 & 0 & 0 & 0 \\
\hline SE & 0 & 0 & 0 & 0 & 3 & 3 \\
\hline SSE & 0 & 0 & 0 & 0 & 0 & 6 \\
\hline S & 0 & 0 & 0 & 0 & 0 & 6 \\
\hline SSW & 0 & 0 & 0 & 0 & 0 & 0 \\
\hline SW & 0 & 0 & 0 & 6 & 0 & $\star$ \\
\hline WSW & 0 & 0 & 0 & 0 & * & * \\
\hline$W$ & 0 & 0 & 3 & * & * & * \\
\hline WNW & 0 & 0 & 0 & * & * & * \\
\hline NW & 0 & 0 & 0 & 0 & * & $\star$ \\
\hline NNW & 0 & 0 & 0 & 0 & 0 & 0 \\
\hline Total & 0 & 0 & 3 & 12 & 9 & 18 \\
\hline
\end{tabular}

Total Population $(0.0-5.0 \mathrm{~km}) 42$

* Sector lies within area apportioned to Falls City, Texas (Susquehanna Western) mill.

A.22 
Site Ray Point, Texas

Latitude $28^{\circ} 31^{\prime} 11^{\prime \prime} \mathrm{N} \quad$ Longitude $98^{\circ} 06^{\prime} 05^{\prime \prime} \mathrm{W}$

Estimated Population - June 1983

Radial Distance $(\mathrm{km})$

\begin{tabular}{|c|c|c|c|c|c|c|}
\hline Direction & $0.0-0.5$ & $\underline{0.5-1.0}$ & $1.0-2.0$ & $2.0-3.0$ & $3.0-4.0$ & $4.0-5.0$ \\
\hline N & 0 & 0 & 0 & 0 & 0 & 0 \\
\hline NNE & 0 & 0 & 3 & 9 & 6 & 14 \\
\hline NE & 0 & 0 & 3 & 3 & 0 & 0 \\
\hline ENE & 0 & 0 & 0 & 3 & 3 & 3 \\
\hline$E$ & 0 & 0 & 6 & 3 & 9 & 6 \\
\hline ESE & 0 & 0 & 9 & 0 & 6 & 0 \\
\hline SE & 0 & 0 & 0 & 0 & 3 & 0 \\
\hline SSE & 0 & 0 & 0 & 3 & 0 & 0 \\
\hline S & 0 & 0 & 0 & 0 & 0 & 0 \\
\hline SSW & 0 & 0 & 0 & 0 & 0 & 0 \\
\hline SW & 0 & 0 & 0 & 0 & 3 & 35 \\
\hline WSW & 0 & 0 & 0 & 0 & 0 & 0 \\
\hline$w$ & 0 & 0 & 0 & 0 & 0 & 0 \\
\hline WNW & 0 & 0 & 0 & 0 & 0 & 0 \\
\hline$N W$ & 0 & 0 & 0 & 0 & 0 & 0 \\
\hline NNW & 0 & 0 & 0 & 0 & 0 & 0 \\
\hline Total & 0 & 0 & 21 & 21 & 30 & 58 \\
\hline
\end{tabular}

Total Population $(0.0-5.0 \mathrm{~km}) 130$

A. 23 
Site Hanksville, Utah

Latitude $37^{\circ} 43^{\prime} 06^{\prime \prime} \mathrm{N} \quad$ Longitude $110^{\circ} 40^{\prime} 51^{\prime \prime} \mathrm{W}$

Estimated Population - June 1983

Radial Distance (km)

$\begin{array}{lcccccc}\text { Direction } & \frac{0.0-0.5}{N} & \frac{0.5-1.0}{1.0-2.0} & \frac{2.0-3.0}{2.0-4.0} & & \frac{4.0-5.0}{3.0} \\ \text { NNE } & 0 & 0 & 0 & 0 & 0 & 0 \\ \text { NE } & 0 & 0 & 0 & 0 & 0 & 0 \\ \text { ENE } & 0 & 0 & 0 & 0 & 0 & 0 \\ \text { E } & 0 & 0 & 0 & 0 & 0 & 0 \\ \text { ESE } & 0 & 0 & 0 & 0 & 0 & 0 \\ \text { SE } & 0 & 0 & 0 & 0 & 0 & 0 \\ \text { SSE } & 0 & 0 & 0 & 0 & 0 & 0 \\ \text { S } & 0 & 0 & 0 & 0 & 0 & 0 \\ \text { SSW } & 0 & 0 & 0 & 0 & 0 & 0 \\ \text { SW } & 0 & 0 & 0 & 0 & 0 & 171 \\ \text { WSW } & 0 & 0 & 0 & 0 & 0 & 0 \\ \text { W } & 0 & 0 & 0 & 0 & 0 & 0 \\ \text { WNW } & 0 & 0 & 0 & 0 & 0 & 0 \\ \text { NW } & 0 & 0 & 0 & 0 & 0 & 0 \\ \text { NNW } & 0 & 0 & 0 & 0 & 0 & 0 \\ \text { Total } & 0 & 0 & 0 & 0 & 0 & 0\end{array}$

Total Population $(0.0-5.0 \mathrm{~km}) 171$

A. 24 
Latitude $47^{\circ} 52^{\prime} 27^{\prime \prime} \mathrm{N} \quad$ Longitude $118^{\circ} 07^{\prime} 00^{\prime \prime} \mathrm{W}$

Estimated Population - June 1983

Radial Distance (km)

\section{Direction}

N

NNE

NE

ENE

E

ESE

SE

SSE

S

SSW

SW

WSW

W

WNW

$\mathrm{NW}$

NNW

Total

$$
\frac{0.0-0.5}{0}
$$$$
0
$$$$
\underline{0.5-1.0}
$$$$
\underline{1.0-2.0}
$$$$
2.0-3.0
$$$$
3.0-4.0
$$$$
\underline{4.0-5.0}
$$

0

0

0

0

0

0

0

0

0

0

0

0

0

0

0

0

0

0

0

0

0

0

0

0

0

0

0

3

6

0

0

0

0

3

0

0

0

0

0

0

0

0

0

0

0

0

0

0

0

3

$0 \quad 0$

0

0

0

0

0

0

3

0

0

0

0

0

0

0

0

5

0

0

0

0

0

5

0

0

0

0

0

0

0

0

0

0

0

32

17

Total Population $(0.0-5.0 \mathrm{~km}) 49$

A. 25 
Site Jeffrey City, Wyoming

Latitude $42^{\circ} 30^{\prime} 32^{\prime \prime} \mathrm{N} \quad$ Longitude $107^{\circ} 47^{\prime} 14^{\prime \prime} \mathrm{W}$

Estimated Population - June 1983

Radial Distance (km)

\begin{tabular}{|c|c|c|c|c|c|c|}
\hline Direction & $0.0-0.5$ & $0.5-1.0$ & $1.0-2.0$ & $2.0-3.0$ & $3.0-4.0$ & $4.0-5.0$ \\
\hline N & 0 & 0 & 0 & 0 & 0 & 0 \\
\hline NNE & 0 & 0 & 0 & 0 & 0 & 0 \\
\hline NE & 0 & 0 & 0 & 0 & 0 & 0 \\
\hline ENE & 0 & 0 & 0 & 0 & 0 & 0 \\
\hline$E$ & 0 & 0 & 0 & 0 & 0 & 0 \\
\hline ESE & 0 & 3 & 0 & 0 & 0 & 0 \\
\hline SE & 0 & 0 & 0 & 21 & 6 & 0 \\
\hline SSE & 0 & 0 & 0 & 3 & 0 & 9 \\
\hline$S$ & 0 & 0 & 0 & 0 & 0 & 0 \\
\hline SSW & 0 & 0 & 0 & 0 & 0 & 0 \\
\hline SW & 0 & 0 & 0 & 0 & 140 & 0 \\
\hline WSW & 0 & 0 & 0 & 0 & 551 & 167 \\
\hline$w$ & 0 & 0 & 0 & 0 & 0 & 0 \\
\hline WNW & 0 & 0 & 0 & 0 & 0 & 0 \\
\hline $\mathrm{NW}$ & 0 & 0 & 0 & 3 & 0 & 0 \\
\hline NNW & 0 & 0 & 0 & 3 & 0 & 0 \\
\hline Total & 0 & 3 & 0 & 30 & 697 & 176 \\
\hline
\end{tabular}

Total Population $(0.0-5.0 \mathrm{~km}) 906$ 
Site Gas Hills, Wyoming (Federal-American Partners)

Latitude $42^{\circ} 47^{\prime} 59^{\prime \prime} \mathrm{N} \quad$ Longitude $107^{\circ} 30^{\prime} 08^{\prime \prime} \mathrm{W}$

Estimated Population - June 1983

Radial Distance $(\mathrm{km})$

\begin{tabular}{|c|c|c|c|c|c|c|}
\hline Direction & $0.0-0.5$ & $0.5-1.0$ & $1.0-2.0$ & $2.0-3.0$ & $3.0-4.0$ & $4.0-5.0$ \\
\hline$N$ & 0 & 0 & 0 & 0 & * & * \\
\hline NNE & 0 & 0 & 0 & * & * & * \\
\hline NE & 0 & 0 & 0 & 0 & * & * \\
\hline ENE & 0 & 0 & 0 & 0 & 0 & * \\
\hline E & 0 & 0 & 0 & 0 & 0 & 0 \\
\hline ESE & 0 & 0 & 0 & 0 & 0 & 0 \\
\hline SE & 0 & 0 & 0 & 0 & 0 & 0 \\
\hline SSE & 0 & 0 & 0 & 0 & 0 & 0 \\
\hline S & 0 & 0 & 0 & 0 & 0 & 0 \\
\hline SSW & 0 & 0 & 0 & 0 & 0 & 0 \\
\hline SW & 0 & 0 & 0 & 0 & 0 & 0 \\
\hline WSW & 0 & 0 & 0 & 0 & 0 & 0 \\
\hline W & 0 & 0 & 0 & 0 & 0 & 0 \\
\hline WNW & 0 & 0 & 0 & 0 & 0 & 0 \\
\hline NW & 0 & 0 & 0 & 0 & 0 & 0 \\
\hline NNW & 0 & 0 & 0 & 0 & 0 & * \\
\hline Total & 0 & 0 & 0 & 0 & 0 & 0 \\
\hline
\end{tabular}

Total Population $(0.0-5.0 \mathrm{~km}) 0$

* Sector lies within area apportioned to Gas Hills, Wyoming (Pathfinder Mines) mill.

A.27 
Latitude $36^{\circ} 55^{\prime} 57^{\prime \prime} \mathrm{N}$ Longitude $109^{\circ} 51^{\prime} 57^{\prime \prime} \mathrm{W}$

Estimated Population - June 1983

Radial Distance $(\mathrm{km})$

\begin{tabular}{|c|c|c|c|c|c|c|}
\hline Direction & $0.0-0.5$ & $0.5-1.0$ & $1.0-2.0$ & $2.0-3.0$ & $3.0-4.0$ & $4.0-5.0$ \\
\hline$N$ & 0 & 0 & 0 & 0 & 4 & 8 \\
\hline NNE & 0 & 0 & 0 & 0 & 4 & 0 \\
\hline NE & 0 & 0 & 0 & 0 & 0 & 0 \\
\hline ENE & 0 & 0 & 0 & 0 & 0 & 0 \\
\hline$E$ & 0 & 4 & 4 & 0 & 0 & 0 \\
\hline ESE & 0 & 0 & 0 & 0 & 0 & 0 \\
\hline SE & 0 & 4 & 0 & 0 & 0 & 0 \\
\hline SSE & 0 & 8 & 8 & 0 & 0 & 0 \\
\hline$S$ & 0 & 4 & 8 & 4 & 0 & 0 \\
\hline SSW & 0 & 0 & 0 & 0 & 0 & 0 \\
\hline SW & 0 & 0 & 0 & 0 & 0 & 0 \\
\hline WSW & 0 & 0 & 0 & 0 & 0 & 0 \\
\hline$W$ & 0 & 0 & 0 & 0 & 0 & 0 \\
\hline WNW & 0 & 0 & 0 & 0 & 0 & 0 \\
\hline NW & 0 & 0 & 0 & 0 & 0 & 0 \\
\hline NNW & 0 & 0 & 0 & 0 & 0 & 0 \\
\hline Total & 0 & 20 & 20 & 4 & 8 & 8 \\
\hline
\end{tabular}

Total Population $(0.0-5.0 \mathrm{~km}) 60$

A. 28 
Site Tuba City, Arizona

Latitude $36^{\circ} 08^{\prime} 41^{\prime \prime} \mathrm{N} \quad$ Longitude $111^{\circ} 08^{\prime} 08^{\prime \prime} \mathrm{W}$

Estimated Population - June 1983

Radial Distance $(\mathrm{km})$

$\begin{array}{lcccccc}\text { Direction } & \underline{0.0-0.5} & \frac{0.5-1.0}{1.0-2.0} & \frac{2.0-3.0}{3.0-4.0} & & \frac{4.0-5.0}{0} \\ \text { N } & 0 & 9 & 0 & 0 & 0 & 0 \\ \text { NNE } & 0 & 0 & 3 & 0 & 0 & 0 \\ \text { NE } & 0 & 0 & 3 & 0 & 0 & 0 \\ \text { ENE } & 0 & 0 & 0 & 6 & 0 & 6 \\ \text { E } & 0 & 0 & 0 & 0 & 0 & 0 \\ \text { ESE } & 0 & 0 & 0 & 3 & 0 & 0 \\ \text { SE } & 0 & 0 & 0 & 0 & 0 & 0 \\ \text { SSE } & 0 & 0 & 0 & 0 & 0 & 0 \\ \text { S } & 0 & 0 & 0 & 0 & 0 & 0 \\ \text { SSW } & 0 & 0 & 0 & 0 & 0 & 0 \\ \text { SW } & 0 & 0 & 0 & 0 & 0 & 0 \\ \text { WSW } & 0 & 0 & 3 & 3 & 0 & 13 \\ \text { W } & 0 & 0 & 0 & 0 & 0 & 0 \\ \text { WNW } & 0 & 0 & 3 & 0 & 0 & 0 \\ \text { NW } & 0 & 0 & 0 & 3 & 0 & 0 \\ \text { NNW } & 0 & 9 & 0 & 0 & 0 & 0 \\ \text { Total } & 0 & 18 & 12 & 15 & 0 & 19\end{array}$

Total Population $(0.0-5.0 \mathrm{~km}) 64$ 
Site Durango, Colorado

Latitude $37^{\circ} 15^{\prime} 55^{\prime \prime} \mathrm{N} \quad$ Longitude $107^{\circ} 53^{\prime} 08^{\prime \prime} \mathrm{W}$

Estimated Population - June 1983

\begin{tabular}{|c|c|c|c|c|c|c|}
\hline \multirow[b]{2}{*}{ Direction } & \multicolumn{5}{|c|}{ Radial Distance $(\mathrm{km})$} & \multirow[b]{2}{*}{$4.0-5.0$} \\
\hline & $\underline{0.0-0.5}$ & $0.5-1.0$ & $1.0-2.0$ & $2.0-3.0$ & $3.0-4.0$ & \\
\hline $\mathrm{N}$ & 0 & 0 & 232 & 928 & 906 & 161 \\
\hline NNE & 0 & 4 & 418 & 1113 & 1278 & 963 \\
\hline NE & 0 & 217 & 954 & 642 & 570 & 726 \\
\hline ENE & 0 & 382 & 920 & 347 & 0 & 0 \\
\hline $\mathrm{E}$ & 0 & 409 & 288 & 0 & 0 & 0 \\
\hline ESE & 0 & 149 & 59 & 0 & 0 & 0 \\
\hline SE & 0 & 0 & 0 & 0 & 0 & 0 \\
\hline SSE & 0 & 0 & 3 & 3 & 0 & 92 \\
\hline$S$ & 0 & 0 & 0 & 0 & 0 & 0 \\
\hline SSW & 0 & 0 & 0 & 0 & 0 & 0 \\
\hline SW & 0 & 0 & 0 & 0 & 3 & 0 \\
\hline WSW & 0 & 0 & 0 & 0 & 3 & 8 \\
\hline W & 0 & 0 & 5 & 16 & 8 & 0 \\
\hline WNW & 0 & 0 & 105 & 0 & 0 & 76 \\
\hline $\mathrm{NW}$ & 0 & 54 & 3 & 3 & 0 & 0 \\
\hline NNW & 3 & 3 & 0 & 0 & 0 & 0 \\
\hline Total & 3 & 1,218 & 2,987 & 3,052 & 2,768 & 2,026 \\
\hline
\end{tabular}

Total Population $(0.0-5.0 \mathrm{~km}) \underline{12,058}$

A. 30 
Latitude $39^{\circ} 02^{\prime} 45^{\prime \prime} \mathrm{N} \quad$ Longitude $108^{\circ} 03^{\prime} 04^{\prime \prime} \mathrm{W}$

Estimated Population - June 1983

\begin{tabular}{|c|c|c|c|c|c|c|}
\hline \multirow[b]{2}{*}{ Direction } & \multicolumn{5}{|c|}{ Radial Distance $(\mathrm{km})$} & \multirow[b]{2}{*}{$4.0-5.0$} \\
\hline & $0.0-0.5$ & $0.5-1.0$ & $1.0-2.0$ & $2.0-3.0$ & $3.0-4.0$ & \\
\hline N & 0 & 0 & 2364 & 1508 & 2392 & 2279 \\
\hline NNE & 0 & 3 & 979 & 842 & 3422 & 1024 \\
\hline NE & 0 & 24 & 106 & 504 & 1631 & 1599 \\
\hline ENE & 0 & 9 & 67 & 524 & 216 & 471 \\
\hline E & 0 & 8 & 62 & 81 & 48 & 118 \\
\hline ESE & 0 & 109 & 575 & 922 & 789 & 320 \\
\hline SE & 9 & 92 & 590 & 334 & 542 & 435 \\
\hline SSE & 3 & 154 & 687 & 191 & 0 & 0 \\
\hline S & 6 & 102 & 820 & 125 & 0 & 0 \\
\hline SSW & 0 & 113 & 424 & 0 & 0 & 0 \\
\hline SW & 0 & 90 & 463 & 0 & 0 & 0 \\
\hline WSW & 0 & 26 & 156 & 40 & 29 & 15 \\
\hline$W$ & 0 & 28 & 13 & 246 & 290 & 399 \\
\hline WNW & 11 & 39 & 15 & 342 & 244 & 481 \\
\hline NW & 0 & 14 & 150 & 465 & 331 & 523 \\
\hline NNW & 0 & 3 & 550 & 1646 & 2155 & 1624 \\
\hline Total & 29 & 814 & 8,021 & 7,770 & 12,089 & 9,288 \\
\hline
\end{tabular}

Total Population $(0.0-5.0 \mathrm{~km}) 38,011$ 
Latitude $38^{\circ} 31^{\prime} 56^{\prime \prime} \mathrm{N}$ Longitude $106^{\circ} 56^{\prime} 30^{\prime \prime} \mathrm{W}$

Estimated Population - June 1983

Radial Distance (km)

\begin{tabular}{|c|c|c|c|c|c|c|}
\hline Direction & $\underline{0.0-0.5}$ & $\underline{0.5-1.0}$ & $1.0-2.0$ & $2.0-3.0$ & $3.0-4.0$ & $4.0-5.0$ \\
\hline N & 0 & 60 & 242 & 80 & 10 & 17 \\
\hline NNE & 0 & 121 & 1090 & 1700 & 481 & 91 \\
\hline NE & 0 & 60 & 607 & 1516 & 62 & 0 \\
\hline ENE & 0 & 0 & 0 & 0 & 0 & 77 \\
\hline$E$ & 0 & 2 & 0 & 0 & 0 & 0 \\
\hline ESE & 0 & 0 & 0 & 0 & 0 & 0 \\
\hline SE & 0 & 0 & 0 & 0 & 0 & 0 \\
\hline SSE & 0 & 0 & 0 & 2 & 2 & 5 \\
\hline S & 0 & 0 & 0 & 59 & 7 & 0 \\
\hline SSW & 0 & 0 & 0 & 2 & 0 & 0 \\
\hline SW & 2 & 2 & 37 & 10 & 0 & 0 \\
\hline WSW & 0 & 27 & 99 & 99 & 15 & 20 \\
\hline$w$ & 5 & 17 & 284 & 40 & 0 & 0 \\
\hline WNW & 0 & 17 & 27 & 12 & 0 & 0 \\
\hline $\mathrm{NW}$ & 0 & 20 & 57 & 2 & 0 & 0 \\
\hline NNW & 0 & 63 & 72 & 90 & 0 & 5 \\
\hline Total & 7 & 389 & 2,515 & 3,612 & 577 & 215 \\
\hline
\end{tabular}

Total Population $(0.0-5.0 \mathrm{~km}) 7315$ 
Site Maybe11, Colorado

Latitude $40^{\circ} 32^{\prime} 36^{\prime \prime} \mathrm{N}$ Longitude $107^{\circ} 59^{\prime} 36^{\prime \prime} \mathrm{W}$

Estimated Population - June 1983

Radial Distance $(\mathrm{km})$

\begin{tabular}{|c|c|c|c|c|c|c|}
\hline Direction & $0.0-0.5$ & $0.5-1.0$ & $1.0-2.0$ & $\underline{2.0-3.0}$ & $3.0-4.0$ & $4.0-5.0$ \\
\hline$N$ & 0 & 0 & 0 & 0 & 0 & 0 \\
\hline NNE & 0 & 0 & 0 & 0 & 0 & 0 \\
\hline NE & 0 & 0 & 0 & 0 & 0 & 0 \\
\hline ENE & 0 & 0 & 0 & 0 & 0 & 0 \\
\hline$E$ & 0 & 0 & 0 & 0 & 0 & 0 \\
\hline ESE & 0 & 0 & 0 & 0 & 0 & 0 \\
\hline SE & 0 & 0 & 0 & 0 & 0 & 0 \\
\hline SSE & 0 & 0 & 0 & 0 & 0 & 0 \\
\hline$S$ & 0 & 0 & 0 & 0 & 0 & 0 \\
\hline SSW & 0 & 0 & 0 & 0 & 0 & 0 \\
\hline SW & 0 & 0 & 0 & 0 & 0 & 0 \\
\hline WSW & 0 & 0 & 0 & 0 & 0 & 0 \\
\hline$w$ & 0 & 0 & 0 & 0 & 0 & 0 \\
\hline WNW & 0 & 0 & 0 & 0 & 0 & 0 \\
\hline $\mathrm{NW}$ & 0 & 0 & 0 & 0 & 0 & 0 \\
\hline NNW & 0 & 0 & 0 & 0 & 0 & 0 \\
\hline Total & 0 & 0 & 0 & 0 & 0 & 0 \\
\hline
\end{tabular}

Total Population $(0.0-5.0 \mathrm{~km}) 0$

A. 33 
Site Naturita, Colorado (General Electric)

Latitude $38^{\circ} 14^{\prime} 30^{\prime \prime} \mathrm{N}$ Longitude $108^{\circ} 36^{\prime} 00^{\prime \prime} \mathrm{W}$

Estimated Population - June 1983

\begin{tabular}{|c|c|c|c|c|c|c|}
\hline \multirow[b]{2}{*}{ Direction } & \multicolumn{4}{|c|}{ Radial Distance $(\mathrm{km})$} & & \multirow[b]{2}{*}{$4.0-5.0$} \\
\hline & $0.0-0.5$ & $0.5-1.0$ & $1.0-2.0$ & $2.0-3.0$ & $3.0-4.0$ & \\
\hline$N$ & 3 & 0 & 0 & 0 & 0 & 0 \\
\hline NNE & 0 & 0 & 0 & 0 & 0 & 9 \\
\hline NE & 0 & 0 & 6 & 3 & 9 & 60 \\
\hline ENE & 0 & 0 & 0 & 3 & 3 & 37 \\
\hline E & 0 & 0 & 0 & 0 & 0 & 0 \\
\hline ESE & 0 & 0 & 0 & 0 & 57 & 352 \\
\hline SE & 0 & 0 & 48 & 14 & 34 & 244 \\
\hline SSE & 0 & 3 & 11 & 0 & * & * \\
\hline S & 0 & 0 & 0 & * & * & * \\
\hline SSW & 0 & 0 & 0 & $\star$ & * & * \\
\hline SW & 0 & 0 & 0 & 0 & * & * \\
\hline WSW & 0 & 0 & 0 & 0 & 0 & * \\
\hline$W$ & 0 & 0 & 0 & 0 & 0 & 0 \\
\hline WNW & 0 & 0 & 0 & 0 & 0 & 0 \\
\hline NW & 0 & 0 & 0 & 0 & 0 & 0 \\
\hline NNW & 0 & 0 & 0 & 0 & 0 & 0 \\
\hline Total & 3 & 3 & 65 & 20 & 103 & 702 \\
\hline
\end{tabular}

Total Population $(0.0-5.0 \mathrm{~km}) 896$

* Sector lies within area apportioned to Naturita, Colorado (Ranchers Exploration) mill. 
Site Naturita, Colorado (Ranchers Exploration)

Latitude $38^{\circ} 12^{\prime} 00^{\prime \prime} \mathrm{N} \quad$ Longitude $108^{\circ} 37^{\prime} 00^{\prime \prime} \mathrm{W}$

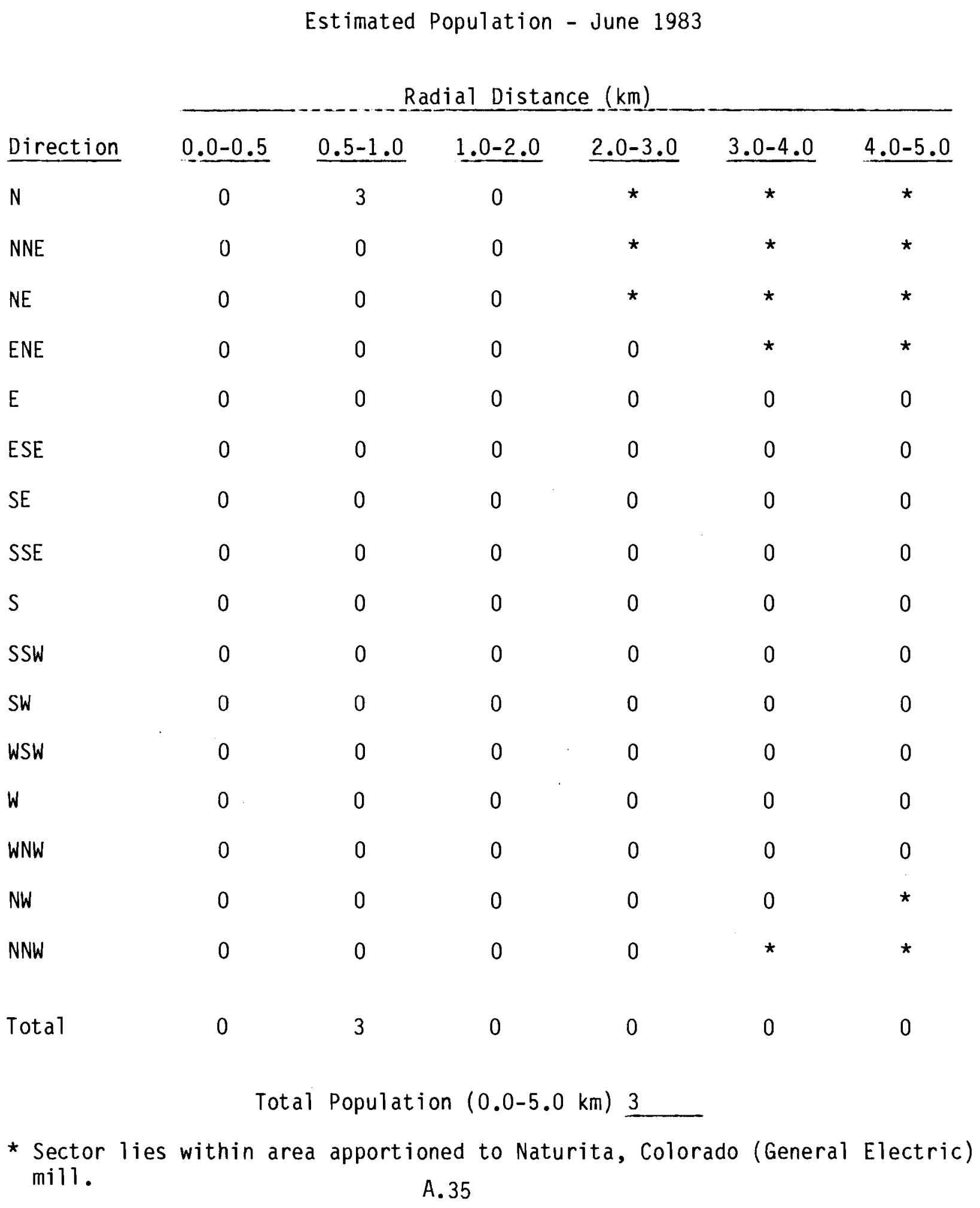


Latitude $39^{\circ} 31^{\prime} 28^{\prime \prime} \mathrm{N} \quad$ Longitude $107^{\circ} 48^{\prime} 49^{\prime \prime} \mathrm{W}$

Estimated Population - June 1983

Radial Distance $(\mathrm{km})$

Direction

$$
\underline{0.0-0.5}
$$$$
\underline{0.5-1.0}
$$$$
\underline{1.0-2.0}
$$$$
2.0-3.0
$$$$
\underline{3.0-4.0}
$$

$\underline{4.0-5.0}$

$\mathrm{N}$

0

3

0

0

0

0

NNE

19

22

3

0

52

3

NE
ENE

0

0

E

ESE

SE

SSE

$S$

SSW

SW

WSW

W

WNW

NW

NNW

Total

19

77

561

400

0

0

0

0

0

0

0

11

3


Site 01d Rifle, Colorado

Latitude $39^{\circ} 31^{\prime} 45^{\prime \prime} \mathrm{N} \quad$ Longitude $107^{\circ} 46^{\prime} 19^{\prime \prime} \mathrm{W}$

Estimated Population - June 1983

Radial Distance (km)

\begin{tabular}{|c|c|c|c|c|c|c|}
\hline Direction & $0.0-0.5$ & $0.5-1.0$ & $1.0-2.0$ & $2.0-3.0$ & $3.0-4.0$ & $4.0-5.0$ \\
\hline N & 0 & 143 & 251 & 127 & 184 & 22 \\
\hline NNE & 0 & 78 & 189 & 11 & 8 & 3 \\
\hline NE & 0 & 5 & 19 & 16 & 5 & 8 \\
\hline ENE & 0 & 0 & 11 & 604 & 24 & 19 \\
\hline$E$ & 0 & 0 & 0 & 3 & 0 & 8 \\
\hline ESE & 0 & 0 & 0 & 0 & 0 & 0 \\
\hline SE & 0 & 0 & 0 & 0 & 0 & 0 \\
\hline SSE & 0 & 11 & 0 & 0 & 0 & 5 \\
\hline$S$ & 0 & 8 & 5 & 0 & 0 & 3 \\
\hline SSW & 0 & 0 & 138 & 0 & 0 & 0 \\
\hline SW & 0 & 22 & 38 & 0 & * & * \\
\hline WSW & 0 & 5 & 3 & 0 & * & * \\
\hline W & 3 & 119 & 197 & * & * & * \\
\hline WNW & 116 & 302 & 772 & 19 & * & * \\
\hline NW & 146 & 375 & 680 & 38 & 0 & 0 \\
\hline NNW & 0 & 138 & 230 & 429 & 119 & 0 \\
\hline Total & 265 & 1206 & 2533 & 1247 & 340 & 68 \\
\hline
\end{tabular}

Total Population $(0.0-5.0 \mathrm{~km}) 5659$

* Sector lies within area apportioned to New Rifle, Colorado mill.

A. 37 
Latitude $38^{\circ} 02^{\prime} 30^{\prime \prime} \mathrm{N} \quad$ Longitude $108^{\circ} 53^{\prime} 30^{\prime \prime} \mathrm{W}$

\begin{tabular}{|c|c|c|c|c|c|c|}
\hline \multirow[b]{2}{*}{ Direction } & & Estimated & $\begin{array}{l}\text { opulation } \\
\text { ial Distar }\end{array}$ & $\begin{array}{l}\text { June } 198 \\
\mathrm{e}(\mathrm{km})\end{array}$ & & \\
\hline & $0.0-0.5$ & $0.5-1.0$ & $1.0-2.0$ & $2.0-3.0$ & $3.0-4.0$ & $4.0-5.0$ \\
\hline N & 0 & 0 & 0 & * & * & * \\
\hline NNE & 0 & 0 & 0 & 0 & 0 & 0 \\
\hline NE & 0 & 0 & 0 & 0 & 0 & 0 \\
\hline ENE & 0 & 0 & 0 & 0 & 0 & 0 \\
\hline E & 0 & 0 & 0 & 0 & 0 & 0 \\
\hline ESE & 0 & 0 & 0 & 0 & 0 & 0 \\
\hline SE & 0 & 0 & 0 & 0 & 0 & 0 \\
\hline SSE & 0 & 0 & 5 & 0 & 0 & 0 \\
\hline S & 0 & 0 & 0 & 0 & 0 & 0 \\
\hline SSW & 0 & 0 & 0 & * & * & * \\
\hline SW & 0 & 0 & * & * & * & * \\
\hline WSW & 0 & * & * & * & * & * \\
\hline$w$ & 0 & * & * & * & * & * \\
\hline WNW & 5 & * & * & * & * & * \\
\hline $\mathrm{NW}$ & 0 & * & * & * & * & * \\
\hline NNW & 0 & 0 & * & * & * & $\star$ \\
\hline Total & 5 & 0 & 5 & 0 & 0 & 0 \\
\hline
\end{tabular}

Total Population $(0.0-5.0 \mathrm{~km}) 10$

* Sector lies within area apportioned to Slick Rock, Colorado (Union Carbide) mill. 
Site Slick Rock, Colorado (Union Carbide)

Lat itude $38^{\circ} 02^{\prime} 43^{\prime \prime} \mathrm{N} \quad$ Longitude $108^{\circ} 54^{\prime} 30^{\prime \prime} \mathrm{W}$

Estimated Population - June 1983

Radial Distance $(\mathrm{km})$

\begin{tabular}{|c|c|c|c|c|c|c|}
\hline Direction & $0.0-0.5$ & $0.5-1.0$ & $1.0-2.0$ & $2.0-3.0$ & $3.0-4.0$ & $4.0-5.0$ \\
\hline N & 0 & 0 & 0 & 0 & 0 & 0 \\
\hline NNE & 0 & 0 & 0 & * & * & * \\
\hline NE & 0 & 0 & * & * & * & * \\
\hline ENE & 0 & 0 & * & * & * & * \\
\hline$E$ & 0 & * & * & * & * & * \\
\hline ESE & 0 & * & * & * & * & * \\
\hline SE & 0 & 0 & * & * & * & * \\
\hline SSE & 0 & 3 & * & * & * & * \\
\hline$S$ & 0 & 0 & 0 & 0 & * & * \\
\hline SSW & 0 & 0 & 0 & 0 & 0 & 0 \\
\hline SW & 0 & 28 & 0 & 0 & 0 & 0 \\
\hline WSW & 0 & 8 & 0 & 0 & 0 & 0 \\
\hline$W$ & 0 & $=0$ & 0 & 0 & 0 & 0 \\
\hline WNW & 0 & 0 & 0 & 0 & 0 & 0 \\
\hline $\mathrm{NW}$ & 0 & 0 & 0 & 0 & 0 & 0 \\
\hline NNW & 0 & 0 & 0 & 0 & 0 & 0 \\
\hline Total & 0 & 39 & 0 & 0 & 0 & 0 \\
\hline
\end{tabular}

Total Population $(0.0-5.0 \mathrm{~km}) 39$

* Sector lies within area apportioned to Slick Rock, Colorado (North Continent) mill.

A. 39 
Latitude $44^{\circ} 05^{\prime} 06^{\prime \prime} \mathrm{N} \quad$ Longitude $115^{\circ} 36^{\prime} 27^{\prime \prime} \mathrm{W}$

Estimated Population - June 1983

Radial Distance $(\mathrm{km})$

\begin{tabular}{|c|c|c|c|c|c|c|}
\hline Direction & $0.0-0.5$ & $0.5-1.0$ & $1.0-2.0$ & $2.0-3.0$ & $3.0-4.0$ & $4.0-5.0$ \\
\hline $\mathrm{N}$ & 0 & 0 & 0 & 0 & 0 & 0 \\
\hline NNE & 0 & 0 & 0 & 0 & 0 & 0 \\
\hline$N E$ & 0 & 0 & 0 & 0 & 0 & 0 \\
\hline ENE & 0 & 0 & 0 & 0 & 0 & 0 \\
\hline$E$ & 0 & 0 & 0 & 0 & 8 & 0 \\
\hline ESE & 0 & 0 & 0 & 0 & 8 & 30 \\
\hline $\mathrm{SE}$ & 0 & 0 & 14 & 0 & 0 & 0 \\
\hline SSE & 0 & 0 & 0 & 0 & 0 & 0 \\
\hline$S$ & 0 & 0 & 0 & 0 & 0 & 0 \\
\hline SSW & 0 & 0 & 0 & 0 & 0 & 0 \\
\hline SW & 3 & 49 & 14 & 0 & 0 & 0 \\
\hline WSW & 0 & 19 & 24 & 0 & 0 & 0 \\
\hline$w$ & 3 & 8 & 35 & 0 & 0 & 0 \\
\hline WNW & 3 & 0 & 0 & 0 & 0 & 0 \\
\hline NW & 0 & 0 & 0 & 0 & 0 & 0 \\
\hline NNW & 0 & 0 & 0 & 0 & 0 & 0 \\
\hline Total & 9 & 76 & 87 & 0 & 16 & 30 \\
\hline
\end{tabular}

Total Population $(0.0-5.0 \mathrm{~km}) 218$
A. 40 
Site Ambrosia Lake, New Mexico (Phillips Petroleum)

Latitude $35^{\circ} 24^{\prime} 34^{\prime \prime} \mathrm{N} \quad$ Longitude $107^{\circ} 47^{\prime} 57^{\prime \prime} \mathrm{W}$

Estimated Population - June 1983

Radial Distance $(\mathrm{km})$

\begin{tabular}{|c|c|c|c|c|c|c|}
\hline Direction & $0.0-0.5$ & $0.5-1.0$ & $1.0-2.0$ & $2.0-3.0$ & $3.0-4.0$ & $4.0-5.0$ \\
\hline$N$ & 0 & 0 & 0 & 0 & 0 & 0 \\
\hline NNE & 0 & 0 & 0 & 0 & 0 & 0 \\
\hline NE & 0 & 0 & 0 & 0 & 0 & 0 \\
\hline ENE & 0 & 0 & 0 & 0 & 0 & 0 \\
\hline$E$ & 0 & 0 & 0 & 0 & 0 & 0 \\
\hline ESE & 0 & 0 & 0 & 0 & 0 & 0 \\
\hline SE & 0 & 0 & 0 & 0 & 0 & 0 \\
\hline SSE & 0 & 0 & 0 & 0 & 0 & 0 \\
\hline S & 0 & 0 & 0 & 0 & 0 & 0 \\
\hline SSW & 0 & 0 & 0 & 0 & * & * \\
\hline SW & 0 & 0 & 0 & $\star$ & * & * \\
\hline WSW & 0 & 0 & 0 & * & * & * \\
\hline W & 0 & 0 & 0 & 0 & * & * \\
\hline WNW & 0 & 0 & 0 & 2 & 0 & * \\
\hline NW & 0 & 0 & 0 & 0 & 0 & 0 \\
\hline NNW & 0 & 0 & 0 & 0 & 0 & 0 \\
\hline Total & 0 & 0 & 0 & 2 & 0 & 0 \\
\hline
\end{tabular}

Total Population $(0.0-5.0 \mathrm{~km}) \underline{2}$

* Sector lies within area apportioned to Ambrosia Lake, New Mexico (Kerr-McGee Nuclear) mill. 
Site Shiprock, New Mexico

Latitude $36^{\circ} 46^{\prime} 11^{\prime \prime} \mathrm{N} \quad$ Longitude $108^{\circ} 41^{\prime} 06^{\prime \prime} \mathrm{W}$

Estimated Population - June 1983

Radial Distance (km)

\begin{tabular}{|c|c|c|c|c|c|c|}
\hline Direction & $0.0-0.5$ & $0.5-1.0$ & $1.0-2.0$ & $2.0-3.0$ & $3.0-4.0$ & $4.0-5.0$ \\
\hline$N$ & 0 & 0 & 246 & 117 & 505 & 369 \\
\hline NNE & 0 & 10 & 143 & 152 & 0 & 6 \\
\hline NE & 0 & 0 & 87 & 463 & 78 & 0 \\
\hline ENE & 0 & 6 & 78 & 84 & 246 & 75 \\
\hline$E$ & 0 & 0 & 10 & 0 & 0 & 0 \\
\hline ESE & 0 & 0 & 0 & 0 & 0 & 0 \\
\hline SE & 0 & 0 & 0 & 0 & 0 & 0 \\
\hline SSE & 0 & 0 & 0 & 0 & 0 & 0 \\
\hline$S$ & 0 & 0 & 6 & 0 & 0 & 0 \\
\hline SSW & 0 & 10 & 3 & 3 & 3 & 0 \\
\hline SW & 0 & 110 & 295 & 62 & 0 & 0 \\
\hline WSW & 0 & 0 & 207 & 49 & 3 & 0 \\
\hline$W$ & 0 & 0 & 382 & 45 & 16 & 0 \\
\hline WNW & 0 & 3 & 162 & 26 & 36 & 0 \\
\hline NW & 0 & 0 & 275 & 10 & 58 & 165 \\
\hline NNW & 0 & 16 & 10 & 23 & 71 & 224 \\
\hline Total & 0 & 155 & 1,904 & 1,034 & 1,016 & 839 \\
\hline
\end{tabular}

Total Population $(0.0-5.0 \mathrm{~km}) 4948$ 
Site Belfield, North Dakota

Latitude $46^{\circ} 52^{\prime} 50^{\prime \prime} \mathrm{N} \quad$ Longitude $103^{\circ} 10^{\prime} 57^{\prime \prime} \mathrm{W}$

Estimated Population - June 1983

Radial Distance $(\mathrm{km})$

\begin{tabular}{|c|c|c|c|c|c|c|}
\hline Direction & $0.0-0.5$ & $0.5-1.0$ & $1.0-2.0$ & $2.0-3.0$ & $3.0-4.0$ & $4.0-5.0$ \\
\hline N & 0 & 0 & 0 & 0 & 0 & 3 \\
\hline NNE & 0 & 0 & 0 & 0 & 0 & 0 \\
\hline$N E$ & 0 & 0 & 0 & 0 & 6 & 0 \\
\hline ENE & 0 & 0 & 0 & 6 & 0 & 3 \\
\hline$E$ & 0 & 0 & 3 & 9 & 3 & 15 \\
\hline ESE & 0 & 0 & 3 & 9 & 0 & 0 \\
\hline SE & 0 & 0 & 0 & 0 & 3 & 3 \\
\hline SSE & 0 & 0 & 0 & 0 & 3 & 0 \\
\hline$S$ & 0 & 0 & 0 & 0 & 0 & 0 \\
\hline SSW & 0 & 3 & 0 & 0 & 3 & 6 \\
\hline SW & 0 & 0 & 0 & 0 & 0 & 6 \\
\hline WSW & 0 & 0 & 0 & 3 & 0 & 0 \\
\hline$W$ & 0 & 3 & 210 & 0 & 0 & 37 \\
\hline WNW & 0 & 0 & 573 & 55 & 0 & 53 \\
\hline NW & 0 & 12 & 387 & 87 & 6 & 6 \\
\hline NNW & 0 & 47 & 15 & 3 & 0 & 0 \\
\hline Total & 0 & 65 & 1,191 & 172 & 24 & 132 \\
\hline
\end{tabular}


Latitude $46^{\circ} 13^{\prime} 01^{\prime \prime} \mathrm{N} \quad$ Longitude $103^{\circ} 32^{\prime} 58^{\prime \prime} \mathrm{W}$

Estimated Population - June 1983

Radial Distance (km)

\begin{tabular}{|c|c|c|c|c|c|c|}
\hline \multirow[b]{2}{*}{ Direction } & & \multirow[b]{2}{*}{$3.0-4.0$} & \multirow[b]{2}{*}{$4.0-5.0$} \\
\hline & $0.0-0.5$ & $0.5-1.0$ & $1.0-2.0$ & $2.0-3.0$ & & \\
\hline$N$ & 0 & 0 & 0 & 0 & 3 & 0 \\
\hline NNE & 0 & 0 & 0 & 0 & 0 & 0 \\
\hline $\mathrm{NE}$ & 0 & 0 & 0 & 0 & 0 & 0 \\
\hline ENE & 0 & 0 & 0 & 0 & 0 & 0 \\
\hline$E$ & 0 & 3 & 0 & 0 & 0 & 0 \\
\hline ESE & 0 & 0 & 0 & 3 & 0 & 0 \\
\hline$S E$ & 0 & 0 & 0 & 0 & 0 & 0 \\
\hline SSE & 0 & 0 & 0 & 0 & 0 & 3 \\
\hline$S$ & 0 & 0 & 0 & 0 & 0 & 0 \\
\hline SSW & 0 & 0 & 3 & 0 & 0 & 0 \\
\hline SW & 0 & 0 & 0 & 0 & 0 & 3 \\
\hline WSW & 0 & 0 & 0 & 0 & 0 & 0 \\
\hline W & 0 & 0 & 0 & 0 & 0 & 0 \\
\hline WNW & 0 & 0 & 0 & 0 & 3 & 6 \\
\hline $\mathrm{NW}$ & 0 & 0 & 0 & 0 & 0 & 0 \\
\hline NNW & 0 & 0 & 6 & 0 & 0 & 0 \\
\hline Total & 0 & 3 & 9 & 3 & 6 & 12 \\
\hline
\end{tabular}

Total Population $(0.0-5.0 \mathrm{~km}) 33$

A. 44 
Site Lakeview, Oregon

Latitude $42^{\circ} 12^{\prime} 34^{\prime \prime} \mathrm{N} \quad$ Longitude $120^{\circ} 22^{\prime} 01^{\prime \prime} \mathrm{W}$

Estimated Population - June 1983

Radial Distance (km)

\begin{tabular}{|c|c|c|c|c|c|c|}
\hline Direction & $0.0-0.5$ & $\underline{0.5-1.0}$ & $1.0-2.0$ & $2.0-3.0$ & $3.0-4.0$ & $4.0-5.0$ \\
\hline N & 0 & 0 & 21 & 19 & 54 & 0 \\
\hline NNE & 0 & 8 & 83 & 0 & 0 & 0 \\
\hline NE & 0 & 0 & 3 & 0 & 0 & 0 \\
\hline ENE & 0 & 0 & 3 & 0 & 0 & 0 \\
\hline$E$ & 0 & 0 & 13 & 0 & 0 & 0 \\
\hline ESE & 0 & 0 & 5 & 0 & 0 & 0 \\
\hline SE & 0 & 0 & 158 & 925 & 257 & 0 \\
\hline SSE & 0 & 0 & 174 & 699 & 1118 & 386 \\
\hline$S$ & 0 & 0 & 64 & 51 & 0 & 0 \\
\hline SSW & 0 & 0 & 3 & 0 & 11 & 8 \\
\hline SW & 0 & 0 & 0 & 5 & 3 & 5 \\
\hline WSW & 0 & 0 & 0 & 0 & 11 & 43 \\
\hline$w$ & 0 & 0 & 0 & 0 & 0 & 11 \\
\hline WNW & 0 & 0 & 3 & 0 & 0 & 8 \\
\hline NW & 0 & 0 & 0 & 0 & 0 & 0 \\
\hline NNW & 0 & 0 & 13 & 5 & 3 & 0 \\
\hline Total & 0 & 16 & 543 & 1704 & 1457 & 464 \\
\hline
\end{tabular}

Total Population $(0.0-5.0 \mathrm{~km}) 4184$ 
Site Canonsburg, Pennsylvania

Latitude $40^{\circ} 15^{\prime} 22^{\prime \prime} \mathrm{N} \quad$ Longitude $80^{\circ} 12^{\prime} 00^{\prime \prime} \mathrm{W}$

Estimated Population - June 1983

Radial Distance (km)

\begin{tabular}{|c|c|c|c|c|c|c|}
\hline Direction & $0.0-0.5$ & $0.5-1.0$ & $1.0-2.0$ & $2.0-3.0$ & $3.0-4.0$ & $4.0-5.0$ \\
\hline N & 87 & 288 & 244 & 129 & 140 & 546 \\
\hline NNE & 88 & 346 & 195 & 236 & 38 & 21 \\
\hline NE & 90 & 346 & 1634 & 2056 & 82 & 38 \\
\hline ENE & 57 & 262 & 1855 & 1382 & 263 & 143 \\
\hline$E$ & 67 & 139 & 496 & 222 & 719 & 400 \\
\hline ESE & 47 & 264 & 859 & 184 & 76 & 94 \\
\hline SE & 89 & 0 & 336 & 120 & 70 & 50 \\
\hline SSE & 152 & 108 & 117 & 35 & 65 & 85 \\
\hline$S$ & 111 & 167 & 12 & 71 & 9 & 30 \\
\hline SSW & 120 & 249 & 38 & 79 & 546 & 525 \\
\hline SW & 29 & 120 & 870 & 360 & 561 & 18 \\
\hline WSW & 0 & 415 & 624 & 15 & 15 & 73 \\
\hline$W$ & 0 & 113 & 413 & 63 & 105 & 102 \\
\hline WNW & 5 & 35 & 149 & 91 & 15 & 30 \\
\hline NW & 5 & 61 & 0 & 62 & 18 & 56 \\
\hline NNW & 3 & 47 & 146 & 21 & 108 & 70 \\
\hline Tota 1 & 950 & 2,960 & 7,988 & 5,126 & 2,830 & 2,281 \\
\hline
\end{tabular}

Total Population $(0.0-5.0 \mathrm{~km}) \underline{22,135}$

A. 46 
Site Falls City, Texas (Susquehanna Western)

Latitude $28^{\circ} 54^{\prime} 16^{\prime \prime} \mathrm{N} \quad$ Longitude $\underline{98^{\circ} 07^{\prime} 40^{\prime \prime} \mathrm{W}}$

Est imated Population - June 1983

\begin{tabular}{|c|c|c|c|c|c|c|}
\hline \multirow[b]{2}{*}{ Direction } & \multicolumn{6}{|c|}{ Radial Distance $(\mathrm{km})$} \\
\hline & $\underline{0.0-0.5}$ & $0.5-1.0$ & $\underline{1.0-2.0}$ & $2.0-3.0$ & $3.0-4.0$ & $4.0-5.0$ \\
\hline N & 0 & 0 & 0 & 0 & 0 & 6 \\
\hline NNE & 0 & 0 & 0 & 0 & 0 & 0 \\
\hline NE & 0 & 0 & 0 & 0 & 0 & * \\
\hline ENE & 0 & 0 & 0 & 0 & * & * \\
\hline$E$ & 3 & 0 & 0 & * & * & * \\
\hline ESE & 0 & 0 & 0 & * & * & * \\
\hline SE & 0 & 0 & 0 & 0 & * & * \\
\hline SSE & 0 & 0 & 0 & 0 & 0 & 0 \\
\hline$S$ & 0 & 0 & 6 & 0 & 0 & 0 \\
\hline SSW & 0 & 0 & 0 & 0 & 0 & 0 \\
\hline SW & 0 & 0 & 0 & 0 & 0 & 0 \\
\hline WSW & 0 & 0 & 0 & 0 & 0 & 0 \\
\hline W & 0 & 0 & 0 & 0 & 0 & 0 \\
\hline WNW & 0 & 0 & 0 & 0 & 0 & 0 \\
\hline NW & 0 & 0 & 6 & 0 & 12 & 3 \\
\hline NNW & 0 & 0 & 6 & 0 & 3 & 0 \\
\hline Total & 3 & 0 & 18 & 0 & 15 & 9 \\
\hline
\end{tabular}

Total Population $(0.0-5.0 \mathrm{~km}) 4 \underline{4}$

* Sector lies within area apportioned to Falls City, Texas (Conoco - Pioneer Nuclear) mill. A. 47 
Latitude $38^{\circ} 58^{\prime} 51^{\prime \prime} \mathrm{N}$ Longitude $110^{\circ} 08^{\prime} 42^{\prime \prime} \mathrm{W}$

\begin{tabular}{|c|c|c|c|c|c|c|}
\hline \multirow[b]{3}{*}{ Direction } & \multicolumn{6}{|c|}{ Estimated Population - June 1983} \\
\hline & \multicolumn{6}{|c|}{ Radial Distance $(\mathrm{km})$} \\
\hline & $\underline{0.0-0.5}$ & $\underline{0.5-1.0}$ & $1.0-2.0$ & $2.0-3.0$ & $3.0-4.0$ & $4.0-5.0$ \\
\hline N & 0 & 0 & 17 & 17 & 17 & 3 \\
\hline NNE & 0 & 0 & 7 & 4 & 0 & 0 \\
\hline NE & 0 & 0 & 0 & 0 & 0 & 0 \\
\hline ENE & 0 & 0 & 0 & 0 & 0 & 0 \\
\hline E & 0 & 0 & 0 & 0 & 0 & 0 \\
\hline ESE & 0 & 0 & 0 & 0 & 0 & 0 \\
\hline SE & 0 & 0 & 0 & 0 & 0 & 0 \\
\hline SSE & 0 & 0 & 0 & 0 & 0 & 0 \\
\hline$S$ & 0 & 0 & 0 & 0 & 0 & 0 \\
\hline SSW & 0 & 0 & 0 & 0 & 0 & 0 \\
\hline SW & 0 & 0 & 3 & 0 & 0 & 0 \\
\hline WSW & 0 & 0 & 0 & 0 & 0 . & 0 \\
\hline W & 0 & 3 & 10 & 0 & 0 & 0 \\
\hline WNW & 0 & 4 & 136 & 233 & 7 & 0 \\
\hline$N W$ & 0 & 7 & 63 & 459 & 136 & 0 \\
\hline NNW & 0 & 0 & 21 & 97 & 237 & 17 \\
\hline Total & 0 & 14 & 257 & 810 & 397 & 20 \\
\hline & To & Populat & $\begin{array}{l}n(0.0-5 \\
\text { A. } 48\end{array}$ & km) 1498 & & \\
\hline
\end{tabular}


Site Mexican Hat, Utah

Latitude $37^{\circ} 08^{\prime} 06^{\prime \prime} \mathrm{N} \quad$ Longitude $109^{\circ} 52^{\prime} 38^{\prime \prime} \mathrm{W}$

Estimated Population - June 1983

Radial Distance $(\mathrm{km})$

\begin{tabular}{|c|c|c|c|c|c|c|}
\hline Direction & $\underline{0.0-0.5}$ & $0.5-1.0$ & $1.0-2.0$ & $2.0-3.0$ & $3.0-4.0$ & $4.0-5.0$ \\
\hline N & 0 & 0 & 0 & 0 & 0 & 0 \\
\hline NNE & 0 & 0 & 0 & 24 & 0 & 0 \\
\hline NE & 0 & 0 & 0 & 77 & 0 & 0 \\
\hline ENE & 0 & 0 & 0 & 0 & 0 & 0 \\
\hline$E$ & 0 & 0 & 0 & 0 & 0 & 0 \\
\hline ESE & 0 & 0 & 0 & 0 & 0 & 0 \\
\hline SE & 0 & 0 & 0 & 0 & 0 & 0 \\
\hline SSE & 0 & 0 & 0 & 0 & 0 & 0 \\
\hline$S$ & 0 & 0 & 0 & 0 & 0 & 0 \\
\hline SSW & 0 & 0 & 0 & 0 & 0 & 0 \\
\hline SW & 0 & 0 & 267 & 0 & 0 & 0 \\
\hline WSW & 0 & 0 & 0 & 0 & 0 & 0 \\
\hline$W$ & 0 & 0 & 4 & 0 & 0 & 0 \\
\hline WNW & 0 & 4 & 8 & 0 & 0 & 0 \\
\hline NW & 0 & 0 & 0 & 0 & 0 & 0 \\
\hline NNW & 0 & 0 & 0 & 0 & 0 & 0 \\
\hline Total & 0 & 4 & 279 & 101 & 0 & 0 \\
\hline
\end{tabular}


Site Salt Lake City, Utah

Latitude $40^{\circ} 42^{\prime} 08^{\prime \prime} \mathrm{N} \quad$ Longitude $111^{\circ} 54^{\prime} 45^{\prime \prime} \mathrm{W}$

Estimated Population - June 1983

Radial Distance $(\mathrm{km})$

\begin{tabular}{|c|c|c|c|c|c|c|}
\hline Direction & $0.0-0.5$ & $0.5-1.0$ & $1.0-2.0$ & $2.0-3.0$ & $3.0-4.0$ & $4.0-5.0$ \\
\hline N & 0 & 0 & 0 & 63 & 439 & 1600 \\
\hline NNE & 0 & 0 & 0 & 20 & 1116 & 2407 \\
\hline NE & 0 & 0 & 12 & 671 & 3702 & 5910 \\
\hline ENE & 0 & 0 & 197 & 2790 & 3061 & 2893 \\
\hline$E$ & 0 & 0 & 551 & 3035 & 2759 & 3381 \\
\hline ESE & 0 & 0 & 251 & 2050 & 3108 & 3713 \\
\hline SE & 0 & 14 & 177 & 533 & 2410 & 3214 \\
\hline SSE & 5 & 14 & 296 & 75 & 479 & 1118 \\
\hline$S$ & 5 & 45 & 65 & 70 & 2871 & 1984 \\
\hline SSW & 0 & 30 & 25 & 228 & 3636 & 1800 \\
\hline SW & 0 & 50 & 599 & 1275 & 1475 & 2440 \\
\hline WSW & 0 & 10 & 350 & 625 & 1172 & 4739 \\
\hline$W$ & 0 & 30 & 114 & 1427 & 2189 & 4301 \\
\hline WNW & 0 & 0 & 170 & 1027 & 461 & 371 \\
\hline NW & 0 & 0 & 115 & 1371 & 111 & 0 \\
\hline NNW & 0 & 0 & 0 & 83 & 1078 & 3092 \\
\hline Total & 10 & 193 & 2,922 & 15,343 & 30,067 & 42,963 \\
\hline
\end{tabular}

Total Population $(0.0-5.0 \mathrm{~km}) \underline{91,498}$ 
Site Converse County, Wyoming

Latitude $43^{\circ} 14^{\prime} 12^{\prime \prime} \mathrm{N} \quad$ Longitude $105^{\circ} 37^{\prime} 13^{\prime \prime} \mathrm{W} \ldots$

Estimated Population - June 1983

Radial Distance $(\mathrm{km})$

\begin{tabular}{|c|c|c|c|c|c|c|}
\hline Direction & $0.0-0.5$ & $0.5-1.0$ & $1.0-2.0$ & $2.0-3.0$ & $3.0-4.0$ & $4.0-5.0$ \\
\hline N & 0 & 0 & 0 & * & * & * \\
\hline NNE & 0 & 0 & 0 & 0 & * & * \\
\hline NE & 0 & 0 & 0 & 0 & 0 & 0 \\
\hline ENE & 0 & 0 & 0 & 0 & 0 & 0 \\
\hline$E$ & 0 & 0 & 0 & 0 & 0 & 0 \\
\hline ESE & 0 & 0 & 0 & 0 & 0 & 0 \\
\hline SE & 0 & 0 & 0 & 0 & 0 & 0 \\
\hline SSE & 0 & 0 & 0 & 0 & 0 & 0 \\
\hline S & 0 & 0 & 0 & 0 & 0 & 9 \\
\hline SSW & 0 & 0 & 0 & 0 & 0 & 0 \\
\hline SW & 0 & 0 & 0 & 9 & 0 & 0 \\
\hline WSW & 0 & 0 & 0 & 0 & 0 & 0 \\
\hline W & 0 & 0 & 0 & 0 & 0 & 0 \\
\hline WNW & 0 & 0 & 0 & 0 & 0 & 0 \\
\hline $\mathrm{NW}$ & 0 & 0 & 0 & 0 & * & * \\
\hline NNW & 0 & 0 & 0 & * & $\star$ & * \\
\hline Total & 0 & 0 & 0 & 9 & 0 & 9 \\
\hline
\end{tabular}

Total Population $(0.0-5.0 \mathrm{~km}) 18$

* Sector lies within area apportioned to Powder River, Wyoming (Rocky Mountain Energy) mil1. 
Latitude $42^{\circ} 59^{\prime} 45^{\prime \prime} \mathrm{N} \quad$ Longitude $108^{\circ} 24^{\prime} 29^{\prime \prime} \mathrm{W} \ldots \ldots$

Estimated Population - June 1983

Radial Distance $(\mathrm{km})$

\begin{tabular}{|c|c|c|c|c|c|c|}
\hline Direction & $0.0-0.5$ & $0.5-1.0$ & $1.0-2.0$ & $2.0-3.0$ & $3.0-4.0$ & $4.0-5.0$ \\
\hline$N$ & 0 & 0 & 0 & 331 & 1234 & 241 \\
\hline NNE & 0 & 6 & 3 & 268 & 1749 & 4050 \\
\hline NE & 0 & 12 & 12 & 3 & 712 & 1639 \\
\hline ENE & 0 & 0 & 18 & 48 & 21 & 0 \\
\hline$E$ & 0 & 9 & 24 & 18 & 12 & 0 \\
\hline ESE & 0 & 6 & 0 & 3 & 9 & 0 \\
\hline SE & 0 & 0 & 0 & 0 & 0 & 0 \\
\hline SSE & 0 & 0 & 0 & 0 & 30 & 0 \\
\hline$S$ & 0 & 0 & 0 & 0 & 0 & 0 \\
\hline SSW & 12 & 0 & 15 & 3 & 0 & 6 \\
\hline SW & 3 & 0 & 24 & 122 & 30 & 6 \\
\hline WSW & 0 & 0 & 27 & 18 & 48 & 113 \\
\hline W & 0 & 3 & 33 & 0 & 6 & 12 \\
\hline WNW & 0 & 6 & 21 & 0 & 0 & 0 \\
\hline NW & 0 & 6 & 15 & 0 & 18 & 298 \\
\hline NNW & 0 & 0 & 0 & 0 & 331 & 104 \\
\hline Total & 15 & 48 & 192 & 814 & 4200 & 6469 \\
\hline
\end{tabular}

Total Population $(0.0-5.0 \mathrm{~km}) \underline{11,738}$ 


\section{DISTRIBUTION}

No. of

Copies

OFFSITE

50 John L. Russell

Guides and Criteria Branch (ANR-460)

U.S. Environmental Protection Agency

Washington, D.C. 20460

27 DOE Technical Information Center

Kathryn McElmury Albrecht

Box 6040

Taos, NM 87571

H. Ward Alter

Terradex Corporation

460 North Wiget Lane

Walnut Creek, CA 94598

Charlie Amato

Arizona Radiation Regulatory Agency

925 South 52nd Street

Tempe, AZ 85281

Niles Andrus

American Nuclear Corporation

P0 Box 2713

Casper, WY 82601

Susan Aranoff

Department of Environmental

Protection

CN 402

Trenton, NJ 08625

Mohamed T. El-Ashry

Tennessee Valley Authority

Knoxville, TN 37902

Atomic Industrial Forum

Attn: Manny Gordon

7101 Wisconsin Avenue

Bethesda, MD 20014
No. of

Copies

OFFSITE

Rick Austermaun

AMAX, Inc.

1707 Cole Boulevard

Golden, C0 80401

Gary Beach

Dept. of Environmental

Quality

State of Wyoming

401 West 19th Street

Cheyenne, WY 82002

Roger Beers

Beers and Dickson

380 Hayes Street, Suite 1

San Francisco, CA 94102

Rosalie Bertell

Ministry of Concern for

Public Health

151 East Street

Buffalo, NY 14207

R.G. Beverly

Union Carbide Corporation

P0 Box 1028

Grand Junction, C0 81502

Bruce Blanchard, Director

Environmental Project Review

U.S. Department of Interior

Washington, D.C. 20240

Gray Bogden

Western Nuclear, Inc.

134 Union Boulevard

Lakewood, C0 80228

V.H. and H.D. Bowers

Route 1, Box 33

Sutherlin, VA 24594 
No. of

Copies

OFFSITE

Carol A. Bratton

Route 3, Box 380

Rochelle, VA 22738

W.H. Budd, Jr. Wyoming Mining Association P0 Box 866

Cheyenne, WY 82003

Linda A. Calhoun

120 Hore Drive

Chesapeake, VA 23320

Luther Carter

4522 Lowell Street, N.W. Washington, D.C. 20016

J.E. Cearley

Conoco, Inc.

PO Box 2197

Houston, TX 77001

Dr. John C. Cobb

University of Colorado

Health Sciences Center

Campus Box C-245

4200 East Ninth Avenue

Denver, CO 80262

J.F. Cochrane

J.R. Simpl ot Company

P0 Box 912

Pocatello, ID 83201

John L. Cole

Route 6, Box 136-H

Chatham, VA 24531

Lewis M. Cook

Chevron Resources Company

595 Market Street

P0 Box 7147

San Francisco, CA 94120
No. of

Copies

OFFSITE

Hattie M. Cooke

831 Hundley Street

Martinsville, VA 24112

Dr. Lyman Coudie

U.S. Environmental Protection Agency

Center for Environmental Research

Cincinnati, $\mathrm{OH} 45268$

Bernard J. Cox

Department of Mines, Queens 1 and

26 th Floor, Mineral House

41-59 George Street

Brisbane, 4000

AUSTRALIA

David B. Crouch

Homestake Mining Company

650 Cal ifornia Street

San Francisco, CA 94108

Janet H. Danavant

1305 Chatham Road

Martinsville, VA 24112

Luke Danielson

Danielson and Euser

1738 Pearl Street

Denver, CO 80203

John Devanney

HFX 7

NCDRH

12720 Twinbrook Parkway

Rockville, MD 20857

Eagle Rock Committee for Survival

Richard Hodg in

535 Los Robles Street

Los Angeles, CA 90041 
No. of

Copies

\section{OFFSITE}

Charlie Ellis

Council of Environment

Room 903

Ninth Street Office Bldg.

Richmond, VA 23219

Environmental Coalition on Nuclear Power

433 Orl ando

State College, PA 16801

Environmental Defense Fund 1657 Pennsylvania Street Denver, CO 80203

Environmental Defense Fund Rocky Mountain Office 1405 Arapahoe Avenue Boulder, C0 80302

Environmental Policy Center 317 Pennsylvania Ave., S.E. Washington, D.C. 20003

Paul Estey

Exxon Mining

PO Box 2180

Houston, TX 77001

Betsy Flick

225 Deerwood Drive

Blairs, VA 24527

Richard H. Folmar

New Mexico Legislative Council 334 State Capital

Sante Fe, NM 87503

Col . David E. Foster

Route 3, Box 147

Gordonsville, VA 22942

Anna Fotias

Legislative Library - U.S. NRC

Room 8717 (MNB)

Washington, D.C. 20555
No. of

Copies

OFFSITE

Friends of the Earth

530 Main Street

Grand Junction, CO 81501

Mayor of Gallup

City of Gallup

Gallup, NM 87301

Bruce S. Garber

P0 Box 8933

Sante Fe, NM 87504

Al Gedricks

Center for Alternative Mining

Development Policy

1121 University Avenue

Madison, WI 53715

Getty 0 iा Company

E.H. Shuler

3810 Wilshire Blvd.

Los Angeles, CA 90010

James C. Gill and

Cl imax Molybdenum Company

1707 Cole Boulevard

Golden, C0 80401

Alan B. Goodman

2405 E. Riviera Drive

Tempe, AZ 85282

Frances Green

National Wildlife Federation

Fleming Law Building

Boulder, C0 80309

C.K. Gunderson

Gunderson $0 i 1$ Company

P0 Box 669

Grants, NM 87020

B.L. Hardy

Rio Algom Corporation

LaSal Route

Moab , UT 84532 
No. of

Copies

OFFSITE

Kathleen Hayden

1245 Chatman Heights

Martinsville, VA 24112

Georgia H. Herbert, Esq.

Piedmont Environmental Council

28-C Main Street

Warrenton, VA 22186

Dr. L.W. Hersl off

Rocky Mountain Energy

10 Longs Peak Drive

Box 2000

Broomfield, CO 80020

Donna Jackson

Process \& Weapons Division

Waste Management Branch

PO Box A

Aiken, SC 29801

Betty Jankus

EP-361, Room $4 \mathrm{G} 057$

U.S. Department of Energy

1000 Independence Ave., S.W.

Washington, D.C. 20585

Chris Jeffers

Rocky Mountain Greenpeace

2029 East 13th Avenue

Denver, CO 80206

Roger A. Johnson

224 South Street

Carlisle, MA 01741

Judith Jolursrud

433 Orlando Avenue

State College, PA 16801

Dr. Carl J. Joluson

42 Hillside Drive

Denver, C0 80215
No. of

Copies

OFFSITE

Tom A. Kennedy

PO Box 3372

Milan, NM 87021

Dr. Daniel Kerlinsky

1001 Yale, N.E.

Albuquerque, 87131

R.C. Kolom

Box 1774

Taos, NM 87571

Richard Krablin

Anaconda Minerals Company

555 Seventeenth Street

Denver, CO 80202

Dr. Barbara Lambert

Route 3, Box 380

Rochelle, VA 22738

Edward Landa

U.S. Geological Survey (MS-413)

Reston, VA 22092

Terry Lash

Deputy Director

Il linois Department of

Nuclear Safety

Springfield, IL 62705

Mary Levy

Route 1, Box 89-AA

New Canton, VA 23123

Plaedra Levy

Taos Environmental Assoc.

Box 4484

Taos, NM 87571

Kathleen E. Lind-Howe

Ebasco Services

$160 \mathrm{Cl}$ ub Avenue

Lyndhurst, NJ 07071 
No. of

Copies

OFFSITE

Louis A. Linkin, Director

Navaho Environmental Protection Administration

Navaho Nation

P0 Box 308

Window Rock, AZ 86515

Harold V. Lovelace

Hal ifax County Planning Commission

PO Box 786

Hal ifax, VA 24558

Angus W. MacDonald

138 East Main Street

Orange, VA 22960

J.P. McCluskey

Cotter Corporation

9305 West Alameda Parkway

Lakewood, C0 80226

Frank McGuire

New Mexicans for Jobs and Energy

236 Montezuma

Sante Fe, M 87501

Dr. A. Magalik

1415 Quincy, N.E.

Albuquerque, NM 87110

Chuck Marshall

JACA Corporation

550 Pinetown Road

Fort Washington, PA 19034

Bruce Martin

Box 207

Al amogordo, NM 88310

Madonna Martin

TVA

Chattanooga, TN 37401
No. of

Copies

OFFSITE

Bob Medlock

Bear Creek Uranium Project

Rocky Mountain Energy

Box 2000

Broomfield, C0 80020

Timothy M. Michel

400 East Water Street

PO Box 157

Charlottesville, VA 22902

Robert R. Mooney

Dept of Social \& Health Sciences

State of Washington

1409 Smith Tower

Seattle, WA 98104

Carl Z. Morgan

1984 Castleway Drive

Atlanta, GA 30345

Mr. Murdock

Pathfinder Mines Corp.

550 California Street

San Francisco, CA 94104

Gary D. Myers

The Fertilizer Institute

1015 18th Street, N.W.

Washington, D.C. 20036

Natural Resources Defense

Council, Inc.

25 Kearny Street

San Francisco, CA 94108

Natural Resources Defense Council, Inc.

1725 I Street, N.W.

Suite 600

Washington, D.C. 20006 
No. of

Copies

OFFSITE

Natural Resources Defense Council, Inc.

122 East 42nd Street

New York, NY 10168

James D. Nel son

S.D. Dept. of Water \& Natural Resources

523 East Capital

Pierre, SD 57501

Eloise F. Nenon

Southside Concerned Citizens

P0 Box 714

Chatham, VA

Jerry B. Nichols

Bldg 9201-3

ORNL

P0 Box $Y$

Oak Ridge, TN 37830

W. and J.R. Oakes

Route 4, Box 78-A

Chatham, VA 24531

Donald R. Ober

134 West Main Street

Orange, VA 22960

Donald Pay

Technical Information Project

P0 Box 682

Pierre, SD 57501

Lawrence Perry

Black Hills Energy Coalition

Rapid City, SD 57701

Walter Perry, I I I

Assistant Attorney General

State of Wyoming

Cheyenne, WY 82002
No. of

Copies

OFFSITE

Steven Pfaff

Getty 0 i 1

P0 Box 2509

Shirley Basin, WY 82615

Chris Platt

Radiation Education Council

P0 Box 705

Lakeview, OR 97630

R.0. Polil

Cornell University

Clark Hall

Ithaca, NY 14853

Political Ecology Research Group

Attn: Katherine Daview

PO Box 14 Martin Scott

Oxford $0 \times 4 \mathrm{HZ}$

England

Dan Predpall

Woodward Clude Consultants

201 Willowbrook Blvd.

Wayne, NJ 07470

James M. Price

Schlegel Lining Technology

P0 Box 7730

The Woodlands, TX 77380

Shelia M. Prindiville Commonwealth of Virginia Council on the Environment Richmond, VA 23219

Anne Rapkin

Environmental Control Section Attorney General's Office Ninth Floor

160 North Lasalle

Chicago, IL 60601 
No. of

Copies

OFFSITE

Anne Rapkin

Environmental Control Section

Attorney General's Office

Ninth Floor

160 North Lasalle

Chicago, IL 60601

Steve and Brenda Reynolds

Route 4, Box 168

Chatham, VA 24531

S. and E. Reynolds

Route 4, Box 77

Chatham, VA 24531

Virginia Reynolds

Box 560

El Prado, NM 87529

George B. Rice

Kerr-McGee Corporation

123 Robert S. Kerr Avenue

Oklahoma City, OK 73125

David C. Ridinger

Newmont Mining Corporation

1090 Vermont Avenue, N.W.

Washington, D.C. 20005

M. I am Ritclue, Chairman

AIF Committee on Mining \& Milling

7101 Wiscons in Avenue

Bethesda, MD 20814

Vern C. Rogers

Rogers \& Assoc. Energy Corp.

515 East 4500 South

Box 330

Salt Lake City, UT 84110

Jim V. Rouse

Envirologic Systems

2425 S. Colorado Blvd

Denver, CO 80222
No. of

Copies

OFFSITE

Donald D. Runnells, PhD

Prof. of Geology and Geochemistry

University of Colorado

Boulder, CO 80301

Mrs. Frederic W. Scott

Bundoran Farm

North Gorden, VA 22959

Lois Sharp

2837 Monroe Street

Falls Church, VA 22042

Dr. Tom Shepard

Water, Waste and Land

1311 South College

Ft. Collins, CO 80524

Earl W. Shortridge

Union Carbide Corporation

PO Box 1029

Grand Junction, C0 81502

W. Darle Shouse

Northwest Environmental

Preservation Commission

280 S. Liberty Street

Winston-Salem, NC 27101

Sierra Club

$\%$ Bruce Hamilton

Northern Great Plains Chapter

P0 Box 1078

Lander, WY 82520

Sierra Club

Rob Smith

Southwest Chapter

615 South 300 East

Salt Lake City, UT 84111

Sierra Club

330 Pennsylvania Ave., S.E.

Washington, D.C. 20003 
No. of

Copies

OFFSITE

Sierra Club Legal Defense

Fund, Inc.

1424 K Street, N.W.

Washington, D.C. 20003

Silver King Mines

Morton Ranch Project

P0 Box 560

Casper, WY 82602

Chris Sl uvey

Southwest Research \& Information Center

P0 Box 4524

Albuquerque, NM 87106

Solar Lobby

1001 Connecticut Ave., N.W.

Suite 510

Washington, D.C. 20036

Southwest Research \&

In formation Center

PO Box 4524

Albuquerque, NM 87106

Mr. James Spahn

NCRP

7910 Woodmont Avenue

Bethesda, MD 20014

Joanne Spangler

444 Downing Drive

Danville, VA 24541

Julie M. Stewart

Pennsylvania Public Interest Coalition

107 Sixth Street, Room 207

Pittsburgh, PA 15222

Robert E. Sundin, Director

Dept. of Environmental Quality

State of Wyoming

401 West 19th Street

Cheyenne, WY 82002
No. of

Copies

OFFSITE

Linda Taylor

Southwest Research \& Information Center

P0 Box 4524

Albuquerque, NM 87106

Techncial Information Project

P0 Box 602

Mandan, ND 58554

James G. Terrill

411 Dundee Drive

Murrysville, PA 15668

Vincent $F$. Tone

3418 Bluehill Avenue

Gallup, M 87301

Joyce Underberg

Sclilegel Corporation

PO Box 23113

Rochester, NY 14692

Mrs. Belva Sours Uppercue

169 High Street

Eastland Hotel, Suite 311

Portl and, ME 04101

Jim Vander

Illinois Attorney General's office

Springfield, IL 62705

Juan Velasques

United Nuclear

P0 Box 8480

Sante Fe, M 87504

Jean-Pierre Villard

Minstone Corporation

2121 South Oneida Street

Suite 400

Denver, C0 80224 
No. of

Copies

OFFSITE

Joseph K. Wagoner

8310 Carrleigh Parkway

Springfield, VA 22152

Mr. Jim Walpole

1875 I. Street, N.W.

Suite 1200

Washington, D.C. 20006

Donald J. Walsh

405 East Mason Avenue

Alexandria, VA 22301

Terry A. Ward

Mary Kathleen Uranium, Ltd.

55 Collins Street

Melbourne, 3000

AUSTRAL IA

Ken Weaver

Colorado State Dept. of Health

1725 12th Avenue

Greeley, C0 80631

Western Colorado Congress

PO Box 472

Montrose, C0 81402

Ivan White

785 S.E. Vista

Gresham, OR 97030

Dr. William H. Wiese

University of New Mexico

School of Medicine

Albuquerque, M 87131

Dale Wilhelm

TVA

Casper, WY 82601

David C. Williams

Americans for Rational

Energy Alternatives

P0 Box 11802

Albuquerque, NM 87102
No. of

Copies

OFFSITE

William Edwin Winn

North Fork Farm

Route 1, Box 419-B

Martinsville, VA 24112

Don Wodek

PO Box 3140

Casper, WY 82602

Ted Wolff

Division 9733

Sandia National Laboratory

Albuquerque, M 87185

Susan Wyatt

Maildrop 13

U.S. Environmental Protection Agency

Research Triangle Park, NC 27711

Louise Yellowman

Coconino County Supervisor

219 East Cherry

Flagstaff, AZ 86001

Michael S. Yesley

Stephenson, Carpenter, Crout \& $01 m s$ ted

PO Box 669

Sante Fe, NM 87504

Roy Young

Sierra Club

Caribou Star Route

Nederl and, C0 80466

Robert E. Yuhnke

Environmental Defense Fund

1405 Arapahoe Avenue

Boulder, C0 80302

Dave Zerwas, Mayor

City of Grants

P0 Box 879

Grants, NM 87020 
PNL -4959

UC -70

No. of

Copies

OFFSITE

Charles Zimmerman

ICAIR Life Systems, Inc. 24755 Highpoint Road

Cleveland, $\mathrm{OH} 44122$

Ms. Phyllis Zitzer

PO Box 761

Pottsdown, PA 19464
No. of

Copies

ONSITE

DOE Richland Operations of fice

HE Ransom

28 Pacific Northwest Laboratory

C.H. Bloomster (5)

D.R. Brown

G.A. Bruno

S.N. Craig

J.A. Dirks (2)

H.D. Freeman

E.A. Griffin

J.W. Reis (3)

J.K. Young

Publishing Coordination (2)

Technical Information (5)

Energy Systems Library (5) 